\title{
1 Basis of narrow-spectrum activity of fidaxomicin on gut pathogen Clostridioides difficile
}

2 Xinyun $\mathrm{Cao}^{1} \uparrow$, Hande Boyaci ${ }^{2} \uparrow$, James $\mathrm{Chen}^{2}, \mathrm{Yu} \mathrm{Bao}{ }^{1}$, Robert Landick ${ }^{1,3^{*}}$, Elizabeth A.

3 Campbell 2* $^{*}$

4 Affiliations:

$5 \quad{ }^{1}$ Department of Biochemistry, University of Wisconsin-Madison; Madison, United States.

$6 \quad{ }^{2}$ Laboratory of Molecular Biophysics, The Rockefeller University; New York, United States.

$7 \quad{ }^{3}$ Department of Bacteriology, University of Wisconsin-Madison; Madison, United States.

$9 \quad \dagger$ These authors contributed equally to this work.

10 *Corresponding authors. Email addresses: campbee@rockefeller.edu, rlandick@wisc.edu

\section{Abstract}

13 Fidaxomicin (Fdx) is widely used to treat Clostridioides difficile (Cdiff) infections (CDIs), but

14 the molecular basis of its narrow-spectrum activity in the human gut microbiome remains

15 enigmatic. CDIs are a leading cause of nosocomial deaths. Fdx, which inhibits RNA polymerase

16 (RNAP), targets Cdiff with minimal effects on gut commensals, reducing CDI recurrence. Here,

17 we present the cryo-electron microscopy structure of Cdiff RNAP in complex with Fdx, allowing

18 us to identify a crucial Fdx-binding determinant of Cdiff RNAP that is absent in most gut

19 microbiota like Proteobacteria and Bacteroidetes. By combining structural, biochemical, and

20 bioinformatic analyses, we establish that a single RNAP residue is a sensitizing element for Fdx

21 narrow-spectrum activity. Our results provide a blueprint for targeted drug design against an

22 important human pathogen. 


\section{Main}

25 Clostridioides difficile (Cdiff) is a gram-positive, spore-forming, and toxin-producing intestinal

26 bacterium that infects the human gut and causes lethal diarrhea (Cdiff infections or CDIs). With

27 the alarming increase in infections caused by highly pathogenic variants, Cdiff has been

28 designated an "urgent threat" by $\mathrm{CDC}^{1}$. Broad-spectrum antibiotics like vancomycin and

29 metronidazole are used to treat CDIs, but these antibiotics decimate the normal gut microbiome,

30 paradoxically priming the gastrointestinal tract to become more prone to CDI recurrences ${ }^{2,3}$

31 (Fig.1a). In 2011, the macrocyclic antibiotic fidaxomicin (Fdx; Fig. 1b) became available to treat

32 CDI. Fdx selectively targets Cdiff but does not effectively kill crucial gut commensals such as

33 Bacteroidetes ${ }^{4}$, abundant microbes in the human gut microbiome that protects against Cdiff

34 colonization ${ }^{5,6}$. Fdx targets the multisubunit bacterial RNA polymerase (RNAP, subunit

35 composition $\left.\alpha_{2} \beta \beta^{\prime} \omega\right)$, which transcribes DNA to RNA in a complex and highly regulated

36 process. However, no structure is available for Clostridial RNAP. Studies using Mycobacterium

37 tuberculosis (Mtb) and Escherichia coli (Eco) RNAPs show that Fdx functions by inhibiting

38 initiation of transcription ${ }^{7-10}$. RNAP forms two mobile pincers that surround DNA ${ }^{11,12}$ and Fdx

39 inhibits initiation by jamming the pincers in an "open" state, preventing one pincer, the clamp,

40 from closing on the DNA. This doorstop-like jamming results in failures both to melt promoter

41 DNA and to secure the DNA in the enzyme's active-site cleft. Although the general architecture

42 of RNAP is similar for all cellular organisms, differences in the primary subunit sequences,

43 peripheral subunits, or lineage-specific insertions that occur in bacterial RNAP ${ }^{13}$ could explain

44 Fdx sensitivity. For example, Mtb RNAP is much more sensitive to Fdx than Eco RNAP ${ }^{7}$, and

45 the essential transcription factor RbpA sensitizes Mtb to Fdx even further ${ }^{7}$. The half-maximal

46 inhibitory concentration or IC50 is $0.2 \mu \mathrm{M}$ for Mtb RNAP with full-length RbpA, $7 \mu \mathrm{M}$ for Mtb

47 RNAP lacking the RbpA-Fdx contacts, and $53 \mu \mathrm{M}$ for Eco RNAP (Extended Data Table 1) ${ }^{7}$.

48 However, Cdiff lacks RbpA leaving the molecular basis of Cdiff sensitivity unresolved.

49 The pathogenicity and limited genetic tools for Cdiff complicate using Cdiff directly for

50 structural and mechanistic studies of RNAP, with a single report of endogenous Cdiff RNAP

51 purification yielding small amounts of enzyme with suboptimal activity ${ }^{14}$. To enable study of 
52 Cdiff RNAP, we created a recombinant system in Eco that yields milligram quantities of Cdiff

53 core RNAP (E) and also enables rapid mutagenesis (see methods and supplement). Cdiff

54 housekeeping $\sigma^{\mathrm{A}}$ factor was also expressed in Eco, purified, and combined with core Cdiff

55 RNAP to produce the holoenzyme $\left(E \sigma^{\mathrm{A}}\right)$. The purity, activity, and yield of $E \sigma^{\mathrm{A}}$ were suitable for

56 structural and biochemical studies (Extended Data Fig. 1a, 1b and 1c).

To visualize the binding of Fdx to its clinical target, we used single particle cryo-electron microscopy (cryo-EM) to solve the structure of Cdiff $\mathrm{E}^{\mathrm{A}}$ in complex with Fdx. We obtained a cryo-EM map representing a single structural class comprising Cdiff $\mathrm{E} \sigma^{\mathrm{A}}$ and bound $\mathrm{Fdx}$ at $3.3 \AA$ nominal resolution, with a local resolution of $\sim 2.7-3 \AA$ around the Fdx-binding pocket (Fig. 1c, Extended Data Figs. 2 and 3, Extended Data Table 2) ${ }^{15}$. The structure reveals key features of the Cdiff $\mathrm{E} \sigma^{\mathrm{A}}$ and provides the first view of a Clostridial $\mathrm{E} \sigma^{\mathrm{A}}$.

Cdiff RNAP contains a lineage-specific insert in the $\beta$ lobe domain that resembles one found in RNAP from Bacillus subtilis (Bsub, a Firmicute like Cdiff), but distinct from the bettercharacterized inserts in Eco RNAP ${ }^{16-18}$. The Firmicute $\beta$ insert corresponds to $\beta \mathrm{i} 5$ identified by sequence analysis ${ }^{13}$ and consists of two copies of the $\beta-\beta^{\prime}$ module 2 (BBM2) protein fold whereas the $\beta$ lobe insert in Eco RNAP occurs at a different position and corresponds to $\beta \mathrm{i} 4$ (Extended Data Fig. 4). Our structure revealed Cdiff ßi5 (4-5 Å; Extended Data Fig. 3) at a position similar to Bsub $\beta \mathrm{i} 5^{17}$ but the Cdiff insert is larger (121 amino acids vs 99 amino acids in Bsub) (Extended Data Fig. 4). The function of the Firmicute $\beta$ i5 is unknown and awaits further study but is unlikely to impact Fdx binding (located $\sim 70 \AA$ away) or activity.

Cdiff $\sigma^{\mathrm{A}}$ possesses all conserved regions of $\sigma$, which were located in the Cdiff E $\sigma^{\mathrm{A}}$ structure

73 at locations similar to those seen for other bacterial housekeeping $\sigma$-factors (Extended Data

74 Fig.5) ${ }^{7,19,20}$. Cryo-EM density was not visible for most of $\sigma$ region 1 (residues $1-115$ of the 150

75 residues in Cdiff region 1), as also seen with other bacterial holoenzymes characterized 76 structurally ${ }^{7,20,21}$. Cdiff $\sigma^{\mathrm{A}}$ lacks the non-conserved region (NCR) insert between regions and 1 77 and 2 found in some other bacteria like Eco (Extended Data Fig. 5) ${ }^{16}$.

78 As seen in other RNAPs ${ }^{7,8,22}$, Fdx appears to stabilize the clamp pincer in an open state, but the Cdiff clamp is twisted slightly toward Fdx relative to the $M t b \mathrm{E} \sigma^{\mathrm{A}}$ structure with $\mathrm{Fdx}$ 
80 (Fig.2a). Opening and closing of RNAP's pincers are required for transcription initiation ${ }^{11,22}$.

81 Fdx binds $M t b$ RNAP at a hinge between two RNAP pincers (the $\beta^{\prime}$ clamp and $\beta$ lobe pincers),

82 thus physically jamming the hinge and locking RNAP in an open conformation that is unable to

83 form a stable initiation complex ${ }^{8,9}$. Fdx occupies the same location in the Cdiff $\mathrm{E} \sigma^{\mathrm{A}}-\mathrm{Fdx}$

84 structure, indicating that the hinge-jamming mechanism is widely conserved. However, the slight

85 twisting of the Cdiff $\beta^{\prime}$ clamp pincer relative to that observed in Fdx-bound Mtb RNAP (Fig. 2a)

86 increases clamp-Fdx contacts (see below).

87 Fdx contacts six key structural components of Cdiff RNAP: $\beta$ clamp, $\beta^{\prime}$ switch region 2

88 (SW2), $\beta$ switch region 3 (SW3), $\beta$ switch region 4 (SW4), $\beta^{\prime}$ zinc-binding-domain (ZBD), and

$89 \quad \beta^{\prime}$ lid (Figs. 2b and 3). We compared the Fdx-binding determinants in Cdiff RNAP to those

90 previously determined in $M t b \operatorname{RNAP}^{7,8}$. Most of the interactions between Fdx and RNAP were

91 conserved between the two species (Extended Data Fig. 6, Extended Data Table 3). In Cdiff

92 RNAP (Mtb numbering in parentheses), Fdx formed direct hydrogen bonds or salt bridges with

93 four residues $\beta$ R1121 (K1101), $\beta^{\prime} \mathrm{K} 84$ (R84), $\beta^{\prime} \mathrm{K} 86$ (K86), and $\beta^{\prime} \mathrm{R} 326$ (R412) and two water-

94 mediated hydrogen bonds with $\beta^{\prime} \mathrm{D} 237$ (E323) and $\sigma \mathrm{H} 294$ (Q434). (Figs. 2b, Extended Data Fig.

956 , and Extended Data Table 3). Fdx binding is also stabilized by a cation- $\pi$ interaction between

96 the $\beta^{\prime}$ R89 and the Fdx macrolide core C3-C5 double bond in both Mtb and Cdiff RNAPs. Cdiff

97 RNAP residues known to confer Fdx-resistance when mutated $(\beta \mathrm{V} 1143 \mathrm{G}, \beta \mathrm{V} 1143 \mathrm{D}, \beta \mathrm{V} 1143 \mathrm{~F}$,

$98 \beta^{\prime} \mathrm{D} 237 \mathrm{Y}$ and $\beta \mathrm{Q} 1074 \mathrm{~K}^{23,24}$ ) were located within $5 \AA$ of Fdx (Extended Data Fig. 6).

99 Of particular interest, $\beta^{\prime} \mathrm{K} 84$ in Cdiff RNAP forms a salt bridge with the oxygen on the

100 phenolic group of Fdx (due to the acidity of phenol) whereas the corresponding residue $\left(\beta^{\prime} \mathrm{R} 84\right)$

101 in $M t b$ forms a cation- $\pi$ interaction with the aromatic ring of the Fdx homodichloroorsellinic acid

102 moiety (Fig. 2b). We propose that these coulombic interactions by $\beta^{\prime} \mathrm{K} 84$ ( $\left.\beta^{\prime} \mathrm{R} 84\right)$ sensitize both

103 RNAPs to tight Fdx binding (see comparison of individual residues in Cdiff and Mtb RNAPs that

104 bind Fdx; Extended Data Figs. 6 and 7, Extended Data Table 3). Mtb RbpA, an essential

105 transcriptional regulator in mycobacteria, lowers the IC50 of Fdx by a factor of 35 via Fdx

106 contacts with two RbpA residues in the N-terminal region ${ }^{7}$. Cdiff RNAP lacks a RbpA homolog,

107 but we observed four hydrophobic interactions between Fdx and Cdiff RNAP (with $\beta$ T1073, 
$\beta^{\prime} \mathrm{M} 319, \beta^{\prime} \mathrm{K} 314$, and $\sigma \mathrm{L} 283$ ) and one water-mediated hydrogen bonding interaction with $\sigma \mathrm{H} 294$ that are not present with the corresponding Mtb RNAP residues (Fig. 2b, Extended Data Fig.6,

110 Extended Data Table 3). Some of these new interactions ( $\beta^{\prime} \mathrm{M} 319$ and $\beta^{\prime} \mathrm{K} 314$ in the clamp) with

111 Fdx are created by the relatively increased rotation of the Cdiff RNAP clamp towards Fdx (Fig.

$1122 \mathrm{a})$.

113 Gram-negative bacteria are more resistant to Fdx than gram-positive bacteria ${ }^{25-27}$. This

114 dichotomy could reflect differences in membrane and cell-wall morphology, differences in

115 RNAPs, or both. To compare the activity of Fdx against Cdiff and Mtb RNAP, we performed

116 abortive transcription assays using purified RNAPs and the native Cdiff rrnC ribosomal RNA

117 promoter (Fig. 2c) ${ }^{14}$. The IC50s of Fdx for Cdiff RNAP E $\sigma^{\mathrm{A}}(\sim 0.2 \mu \mathrm{M})$ and $M t b$ E $\sigma^{\mathrm{A}}$ including

$118 \operatorname{RbpA}(\sim 0.3 \mu \mathrm{M})$ are similar, consistent with our structural observations (Fig. 2d, Extended Data

119 Fig. 6). These IC50s are two orders of magnitude lower than that for E. coli $\sigma^{70}$-holoenzyme

$120\left(\mathrm{E}^{70}\right)$ on the same DNA template (Fig. 2d, Extended Data Fig. 8), suggesting that the

121 differences in RNAPs contribute significantly to the differences in MICs between Cdiff (a gram-

122 positive) and gram-negative bacteria. This observation suggests that the Fdx-binding residues

123 identified in Mtb and Cdiff RNAPs can be used as a reference to predict Fdx potency in other

124 bacterial species, including gut commensals ${ }^{25}$.

125 We next used our Mtb and Cdiff RNAP-Fdx structures to predict the interactions responsible

126 for the narrow spectrum activity of Fdx. Using sequence alignments of $\beta^{\prime}$ and $\beta$ from bacterial

127 species with reported Fdx MICs ${ }^{25,27}$ (Extended Data Fig. 9), we found that the Fdx-binding

128 residues identified in $M t b$ and Cdiff RNAP are mostly conserved among these divergent bacteria,

129 except for the aforementioned $\beta^{\prime} \mathrm{K} 84$ ( $\beta^{\prime} \mathrm{R} 84$ in $\left.M t b\right)$ and $\beta^{\prime} \mathrm{S} 85$ ( $\beta^{\prime} \mathrm{A} 85$ in $\left.M t b\right) . \beta^{\prime} \mathrm{K} 84$ and S85

130 are located in the ZBD. $\beta^{\prime} \mathrm{K} 84$ forms a salt bridge with the likely ionized Fdx O13 whereas S85

$131 \mathrm{C} \beta$ forms a nonpolar interaction with the Fdx C32 methyl group (Figs. 1b, 3 and Extended Data

132 Fig. 6, Extended Data Table 3). We focused on $\beta^{\prime} \mathrm{K} 84$ because all species contain a $\mathrm{C} \beta$ at

133 position 85 whereas position 84 displays an intriguingly divergent pattern among gut commensal

134 bacteria (Extended Data Fig. 9). For gram-positive bacteria, which are hypersensitive to Fdx

135 (MIC $<0.125 \mu \mathrm{g} / \mathrm{mL}$ ), the $\beta^{\prime} \mathrm{K} 84$ position is always positively charged ( $\mathrm{K}$ or $\mathrm{R}$ ). However, for 
136 gram-negative bacteria, which are resistant to $\mathrm{Fdx}(\mathrm{MIC}>32 \mu \mathrm{g} / \mathrm{mL}), \beta^{\prime} \mathrm{K} 84$ is replaced by a

137 neutral residue (Q in Eco or L in Pseudomonas aeruginosa and Neisseria meningitidis; Extended

138 Data Fig. 9). Notably, in Bacteroidetes, which are highly resistant to Fdx (MIC >32 $\mu$ g/mL, e.g.,

139 Bacteroides uniformis, Bacteroides ovatus, and Bacteroides distasonis), $\beta^{\prime} \mathrm{K} 84$ is replaced by

140 negatively charged glutamic acid (E). In an analysis of common species present in the human gut

141 microbiota (Extended Data Table 4) ${ }^{28,29}, \beta^{\prime} \mathrm{K} 84$ is replaced by E in Bacteroidetes (the most

142 abundant bacteria ${ }^{30,31}$ ) and by neutral residues (Q, T, or S) in Proteobacteria (Figs. 4a, 4b, and

143 Extended Data Fig.10). We thus refer to $\beta^{\prime} \mathrm{K} 84$ as the Fdx sensitizer and propose it is crucial for

144 tight Fdx binding in two ways: first, by forming a salt bridge (a proton-mediated ionic

145 interaction) between the positively charged $\varepsilon$-amino group of $\beta^{\prime} \mathrm{K} 84$ and a negatively charged

146 phenolic oxygen of Fdx; and second, by rigidifying the $\alpha$-helix of the ZBD and thus facilitating

147 backbone hydrophobic and hydrogen-bonding interactions with downstream residues S85 (A85

148 in Mtb) and K86 (Fig. 3 and Extended Data Figs. 6 and 7).

149 We hypothesized that variation in the Fdx sensitizer plays a key role in determining the 150 potency of Fdx activity on RNAP from different clades. To test this hypothesis, we constructed 151 substitutions $\beta^{\prime} \mathrm{K} 84 \mathrm{E}, \beta^{\prime} \mathrm{K} 84 \mathrm{Q}$, and $\beta^{\prime} \mathrm{K} 84 \mathrm{R}$ in Cdiff RNAP and $\beta^{\prime} \mathrm{Q} 94 \mathrm{~K}$ in Eco RNAP and then

152 compared their inhibition by Fdx using the Cdiff rrnC abortive initiation assay (Figs. 4c, 4d,

153 Extended Data Figs. 11, and 12). Fdx inhibits Cdiff wild-type (WT), $\beta^{\prime} \mathrm{K} 84 \mathrm{Q}$, and $\beta^{\prime} \mathrm{K} 84 \mathrm{R}$

154 RNAPs at similar sub- $\mu \mathrm{M}$ concentrations. However, inhibition of Cdiff $\beta^{\prime} \mathrm{K} 84 \mathrm{E}$ RNAP requires a

155 ten-fold higher concentration of Fdx than WT, indicating greater resistance to Fdx (Fig. 4c and

156 Extended Data Fig.11). This result is consistent with our hypothesis that the negatively charged

157 carboxyl group on the side chain of $\beta^{\prime} \mathrm{K} 84 \mathrm{E}$ repels to the negative oxygen of $\mathrm{Fdx}$ and disrupts the 158 polar interaction, whereas the $\beta^{\prime} \mathrm{K} 84 \mathrm{Q}$ must be less disruptive to Cdiff RNAP-Fdx interaction

159 possibly because the glutamine is capable of forming a hydrogen bond with Fdx.

160 To test the effect of positive versus neutral charge at the Fdx sensitizer in the context of an 161 RNAP that is relatively Fdx-resistant, we compared WT and $\beta^{\prime} \mathrm{Q} 94 \mathrm{~K}$ Eco RNAPs. The Eco

$162 \quad \beta^{\prime} \mathrm{Q} 94 \mathrm{~K}$ substitution endows a positive charge at the position and dramatically increases

163 sensitivity to Fdx (the IC50 decreased by a factor of 20; Fig. 4d, Extended Data Fig. 12). This 
164 result indicates that the lack of positive charge at the sensitizer position is indeed a crucial

165 contributor to resistance to Fdx in Proteobacteria and posed an interesting discrepancy with the

166 lack of effect of the Cdiff $\beta^{\prime}$ K84Q substitution. We hypothesize that other differences between

167 Cdiff and Eco RNAP such as the relative flexibilities of the ZBD and clamp allow Cdiff RNAP,

168 but not Eco RNAP, to sustain stronger interactions when the key position is neutral (Q).

169 However, when a negative charge (E) is present at the sensitizer position, the repulsion between

170 the carboxylic side chain (present in Bacteroidetes) and the Fdx phenolic oxygen leads to Fdx-

171 resistance. The increased susceptibility of Eco $\beta^{\prime} \mathrm{Q} 94 \mathrm{~K}$ RNAP to Fdx confirms that the

172 positively-charged sensitizer (as found in Cdiff and Mtb) is crucial for conferring Fdx sensitivity.

173 In summary, our high-resolution structure of Cdiff RNAP $\sigma^{\mathrm{A}}$ reveals features that are likely

174 to be specific to Clostridia and, to some extent Firmicutes and gram-positive bacteria. Analysis

175 of this structure, in combination with bioinformatics and structure-guided functional assays,

176 revealed a "sensitizing" determinant for Fdx, which turns out to be a single amino-acid residue in

177 the ZBD of the RNAP $\beta$ ' subunit. This work sheds light on how Fdx selectively targets Cdiff

178 versus beneficial gut commensals like Bacteroidetes. Although wide-spectrum antibiotics are

179 broadly effective therapies, our results highlight the advantages of narrow-spectrum antibiotics to

180 treat intestinal infections and likely other bacterial infections. Treatment by narrow-spectrum

181 antibiotics would reduce widespread antibiotic resistance and reduce the side effects caused by

182 the collateral eradication of the beneficial bacteria in the gut microbiome. Using a similar

183 approach to that applied here, further elucidation of diverse bacterial RNAP structures and

184 mechanisms can provide a blueprint for designer antibiotics that leverage natural microbial

185 competition to combat pathogens more effectively.

\section{References}

1881 (https://www.cdc.gov/drugresistance/pdf/threats-report/2019-ar-threats-report-508.pdf, 2019).

1892 Louie, T. J. et al. Fidaxomicin versus vancomycin for Clostridium difficile infection. N Engl J Med 364,

190 422-431, doi:10.1056/NEJMoa0910812 (2011).

1913 Crawford, T., Huesgen, E. \& Danziger, L. Fidaxomicin: a novel macrocyclic antibiotic for the treatment of

192

193

194 Clostridium difficile infection. Am J Health Syst Pharm 69, 933-943, doi:10.2146/ajhp110371 (2012).

4 Louie, T. J., Emery, J., Krulicki, W., Byrne, B. \& Mah, M. OPT-80 eliminates Clostridium difficile and is sparing of bacteroides species during treatment of C. difficile infection. Antimicrob Agents Chemother 53, 261-263, doi:10.1128/AAC.01443-07 (2009). 
Lee, Y. J. et al. Protective Factors in the Intestinal Microbiome Against Clostridium difficile Infection in Recipients of Allogeneic Hematopoietic Stem Cell Transplantation. J Infect Dis 215, 1117-1123, doi:10.1093/infdis/jix011 (2017).

6 Vincent, C. \& Manges, A. R. Antimicrobial Use, Human Gut Microbiota and Clostridium difficile Colonization and Infection. Antibiotics (Basel) 4, 230-253, doi:10.3390/antibiotics4030230 (2015).

7 Boyaci, H. et al. Fidaxomicin jams Mycobacterium tuberculosis RNA polymerase motions needed for initiation via RbpA contacts. Elife 7, doi:10.7554/eLife.34823 (2018).

8 Lin, W. et al. Structural Basis of Transcription Inhibition by Fidaxomicin (Lipiarmycin A3). Mol Cell 70, 60-71 e15, doi:10.1016/j.molcel.2018.02.026 (2018).

9 Morichaud, Z., Chaloin, L. \& Brodolin, K. Regions 1.2 and 3.2 of the RNA Polymerase sigma Subunit Promote DNA Melting and Attenuate Action of the Antibiotic Lipiarmycin. J Mol Biol 428, 463-476, doi:10.1016/j.jmb.2015.12.017 (2016).

10 Tupin, A., Gualtieri, M., Leonetti, J. P. \& Brodolin, K. The transcription inhibitor lipiarmycin blocks DNA fitting into the RNA polymerase catalytic site. EMBO J 29, 2527-2537, doi:10.1038/emboj.2010.135 (2010).

11 Boyaci, H., Chen, J., Jansen, R., Darst, S. A. \& Campbell, E. A. Structures of an RNA polymerase promoter melting intermediate elucidate DNA unwinding. Nature 565, 382-385, doi:10.1038/s41586-0180840-5 (2019).

12 Chen, J., Boyaci, H. \& Campbell, E. A. Diverse and unified mechanisms of transcription initiation in bacteria. Nat Rev Microbiol 19, 95-109, doi:10.1038/s41579-020-00450-2 (2021).

13 Lane, W. J. \& Darst, S. A. Molecular evolution of multisubunit RNA polymerases: sequence analysis. $J$ Mol Biol 395, 671-685, doi:10.1016/j.jmb.2009.10.062 (2010).

14 Mani, N., Dupuy, B. \& Sonenshein, A. L. Isolation of RNA polymerase from Clostridium difficile and characterization of glutamate dehydrogenase and rRNA gene promoters in vitro and in vivo. $J$ Bacteriol 188, 96-102, doi:10.1128/JB.188.1.96-102.2006 (2006).

15 Cardone, G., Heymann, J. B. \& Steven, A. C. One number does not fit all: mapping local variations in resolution in cryo-EM reconstructions. J Struct Biol 184, 226-236, doi:10.1016/j.jsb.2013.08.002 (2013). (ing of Escherichia coli RNA polymerase involves misappropriation of sigma70 domain 1.1. Proc Natl Acad Sci U S A 110, 19772-19777, doi:10.1073/pnas.1314576110 (2013). Pei, H. H. et al. The delta subunit and NTPase HelD institute a two-pronged mechanism for RNA polymerase recycling. Nat Commun 11, 6418, doi:10.1038/s41467-020-20159-3 (2020).

Fang, C. et al. The bacterial multidrug resistance regulator BmrR distorts promoter DNA to activate transcription. Nat Commun 11, 6284, doi:10.1038/s41467-020-20134-y (2020). Campbell, E. A. et al. Structure of the bacterial RNA polymerase promoter specificity sigma subunit. Mol Cell 9, 527-539, doi:10.1016/s1097-2765(02)00470-7 (2002).
Hubin, E. A et al. Structure and function of the mycobacterial tr. essential regulator RbpA. Elife 6, doi:10.7554/eLife.22520 (2017).

Chen, J. et al. 6S RNA Mimics B-Form DNA to Regulate Escherichia coli RNA Polymerase. Mol Cell 68, 388-397 e386, doi:10.1016/j.molcel.2017.09.006 (2017).

Feklistov, A. et al. RNA polymerase motions during promoter melting. Science 356, 863-866, doi:10.1126/science.aam7858 (2017).
Babakhani, F., Seddon, J. \& Sears, P. Cor fidaxomicin and the rifamycins in Clostidiutive microbiological studies of transcription inhibitors doi:10.1128/AAC.02572-13 (2014).

Kuehne, S. A. et al. Characterization of the impact of rpoB mutations on the in vitro and in vivo competitive fitness of Clostridium difficile and susceptibility to fidaxomicin. $J$ Antimicrob Chemother $\mathbf{7 3}$, 973-980, doi:10.1093/jac/dkx486 (2018).

25 Goldstein, E. J., Babakhani, F. \& Citron, D. M. Antimicrobial activities of fidaxomicin. Clin Infect Dis 55 Suppl 2, S143-148, doi:10.1093/cid/cis339 (2012). Kurabachew, M. et al. Lipiarmycin targets RNA polymerase and has good activity against multidrugresistant strains of Mycobacterium tuberculosis. J Antimicrob Chemother 62, 713-719, doi:10.1093/jac/dkn269 (2008).

27 Srivastava, A. et al. New target for inhibition of bacterial RNA polymerase: 'switch region'. Curr Opin Microbiol 14, 532-543, doi:10.1016/j.mib.2011.07.030 (2011).
Forster, S. C. et al. A human gut bacterial genome and culture coll analyses. Nat Biotechnol 37, 186-192, doi:10.1038/s41587-018-0009-7 (2019). 
25229 Qin, J. et al. A human gut microbial gene catalogue established by metagenomic sequencing. Nature 464, 59-65, doi:10.1038/nature08821 (2010).

30 Wexler, A. G. \& Goodman, A. L. An insider's perspective: Bacteroides as a window into the microbiome. Nat Microbiol 2, 17026, doi:10.1038/nmicrobiol.2017.26 (2017).

31 King, C. H. et al. Baseline human gut microbiota profile in healthy people and standard reporting template. PLoS One 14, e0206484, doi:10.1371/journal.pone.0206484 (2019). 

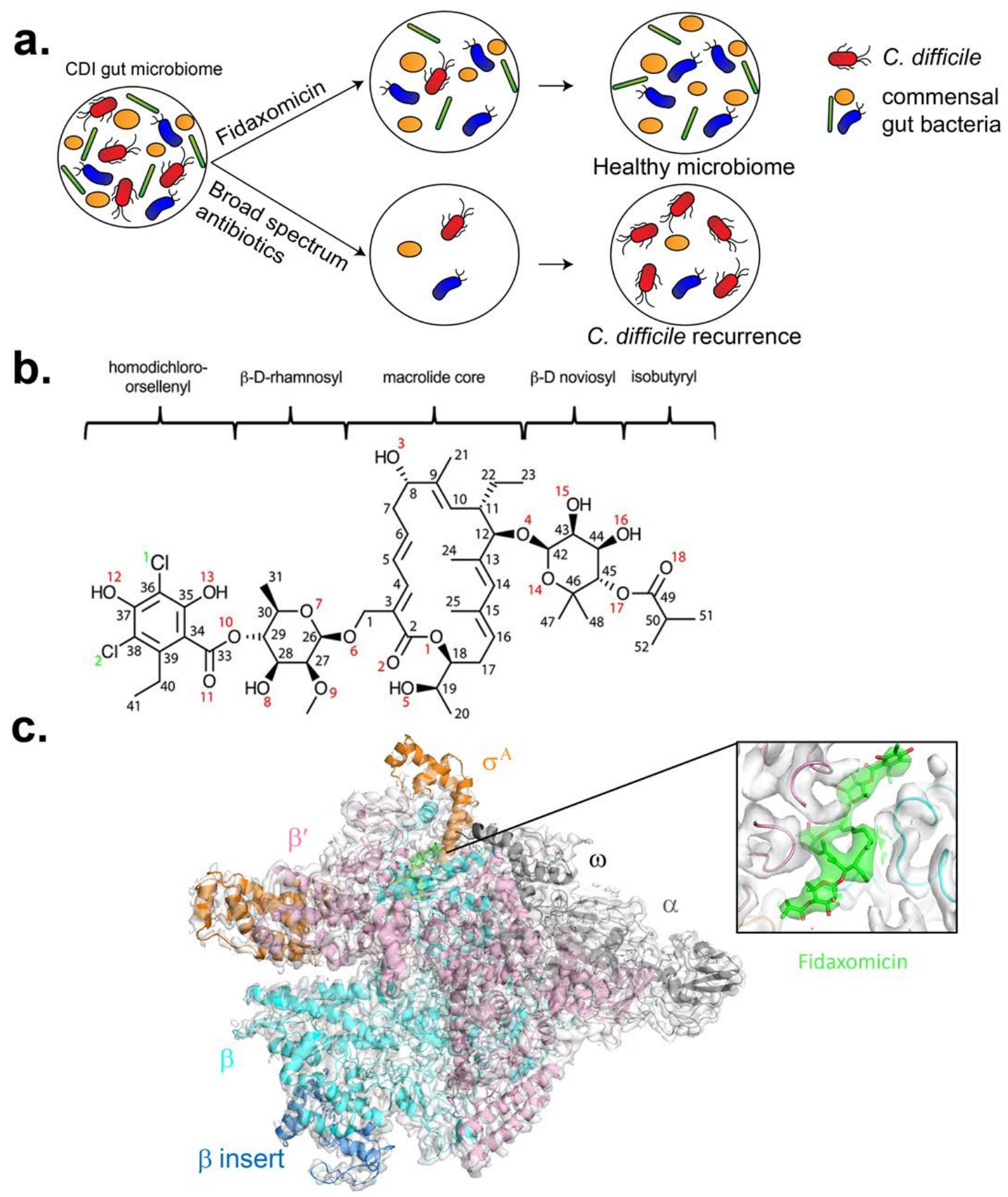

263 Fig. 1. Fidaxomicin is a narrow-spectrum antimicrobial that inhibits RNAP. a, Diagram illustrating how fidaxomicin specifically targets Cdiff without affecting gut commensals and thus reduces recurrence (upper circles). For CDI patients treated with broad-spectrum antibiotics (lower circles), the abundance of gut commensals drops simultaneously with Cdiff resulting in high rates of Cdiff recurrence. b, Chemical structure of Fdx. c, Cryo-EM structure of Cdiff E $\sigma^{\mathrm{A}}$ in complex with Fdx. The $E \sigma^{\mathrm{A}}$ model is colored by subunits according to the key, and the $3.26 \AA$ cryo-EM map is represented in a white transparent surface. The cryo-EM density for Fdx is shown in the inset as a green transparent surface. 


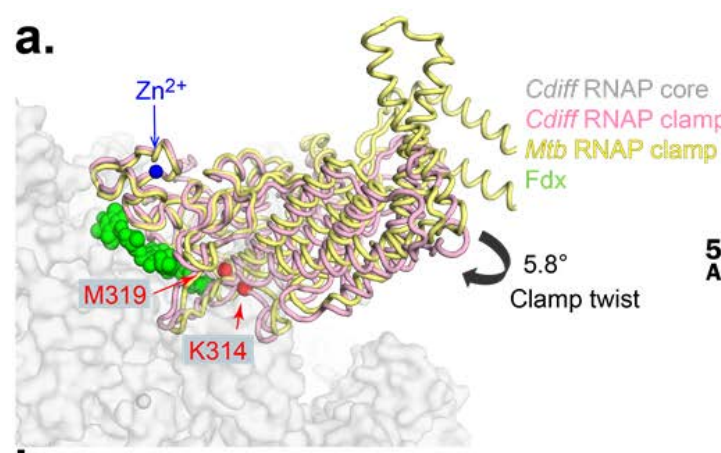

b.

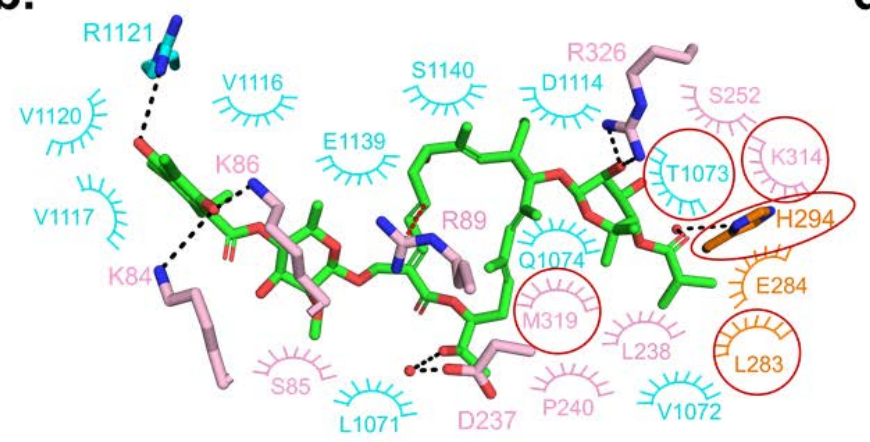

c. C. difficile $r r n C$

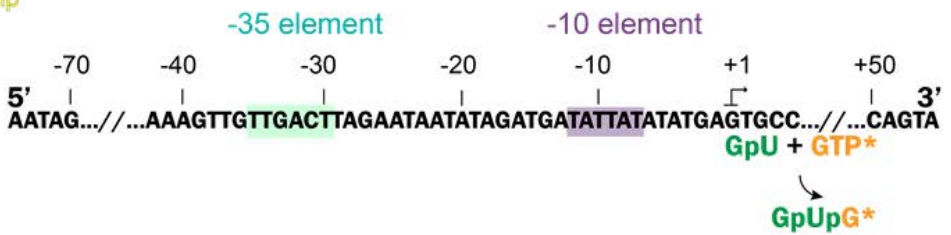

d.

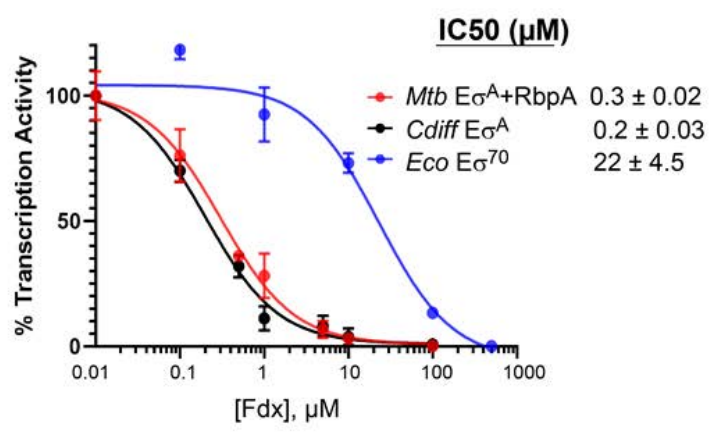

Fig. 2. Fdx binding and inhibition of the Cdiff $\mathrm{E} \sigma^{\mathrm{A}}$ a, Clamp differences between $C$ diff and $M t b$ RNAP. The Cdiff RNAP clamp (pink) is twisted (5.8 $8^{\circ}$ towards Fdx compared with the $M t b$ RNAP-Fdx clamp (yellow) (PDB ID: 6BZO). The actinobacterial specific insert in the clamp is partially cropped. The clamp residues, $\beta^{\prime} \mathrm{K} 314$ and $\beta^{\prime} \mathrm{M} 319$, that interact with $\mathrm{Fdx}$ (shown in green spheres) in Cdiff but not Mtb RNAP are shown as red spheres. The zinc in the ZBD is shown as a blue sphere. b, The interactions between Fdx and Cdiff RNAP are shown. Hydrogenbonding interactions are shown as black dashed lines. The cation- $\pi$ interaction of $\beta^{\prime} \mathrm{R} 89$ is shown with a red dashed line. Arches represent hydrophobic interactions. RNAP residues are colored corresponding to subunits: cyan $(\beta)$ and pink $\left(\beta^{\prime}\right)$. The Fdx-contacting residues that are not present in the $M t b E \sigma^{\mathrm{A}}-\mathrm{Fdx}$ structure (pdb 6BZO) are marked with red circles. $\mathbf{c}$, The sequence of the native Cdiff ribosomal RNAP rrnC promoter used in the in vitro transcription assay in $\mathbf{d}$. The -10 and -35 promoter elements are shaded in purple and green, respectively. The abortive transcription reaction used to test Fdx effects is indicated below the sequence $\left(*,\left[\alpha-{ }_{-}^{32} \mathrm{P}\right] \mathrm{GTP}\right.$ used to label the abortive product $\mathrm{GpUpG})$. d, Fdx inhibits $C$ diff $E \sigma^{\mathrm{A}}$ and $M t b \operatorname{RbpA}-\mathrm{E} \sigma^{\mathrm{A}}$ similarly and $\sim 100$ times more effectively than $E c o \mathrm{E}^{70}$. Error bars are standard deviations (SD) of three independent replicates (for some points, SD was smaller than the data symbols). 


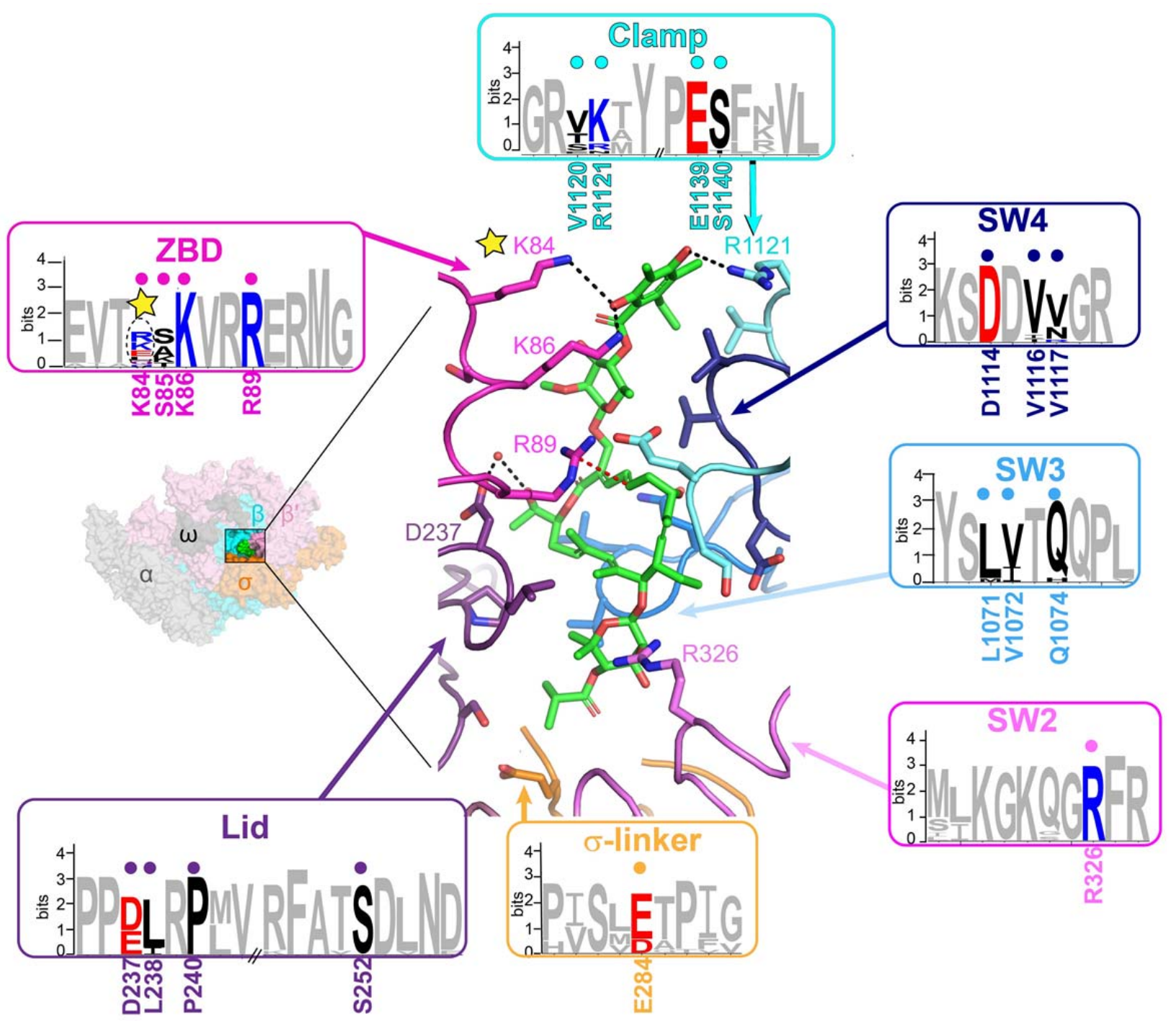

Fig. 3. Analysis of Fdx-interacting residues across bacterial lineages. Cdiff RNAP E $\sigma^{\mathrm{A}}-\mathrm{Fdx}$ is shown as a molecular surface for orientation (left inset). The boxed region is magnified on the right with RNAP subunits shown as $\alpha$-carbon backbone worms, Fdx-interacting residues conserved between Mtb and Cdiff shown as side-chain sticks, and Fdx (green) shown as sticks. Non-carbon atoms are red (oxygen) and blue (nitrogen). The Mtb-Cdiff-conserved, Fdxinteracting residues shown in the cartoon structure are labeled under the sequence logos. Amino acids that make hydrophilic interactions with $\mathrm{Fdx}$ are labeled on the structure. Representative bacterial species with published Fdx MICs were used to make the logos ${ }^{25}$. See Extended Data Fig. 9 for detailed sequence alignments. Most Fdx-interacting residues are conserved except residues corresponding to Cdiff $\beta^{\prime}$ S 85 and the sensitizer (yellow star corresponding to Cdiff $\beta^{\prime} \mathrm{K} 84$ ). 
a. Relative abundance of human gut microbiota species and the

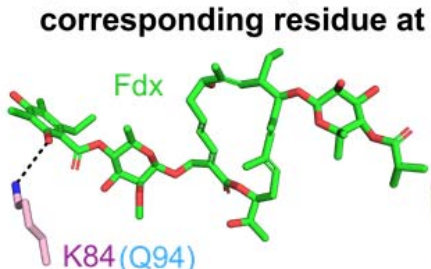

Bacteroidetes

Firmicutes

C.

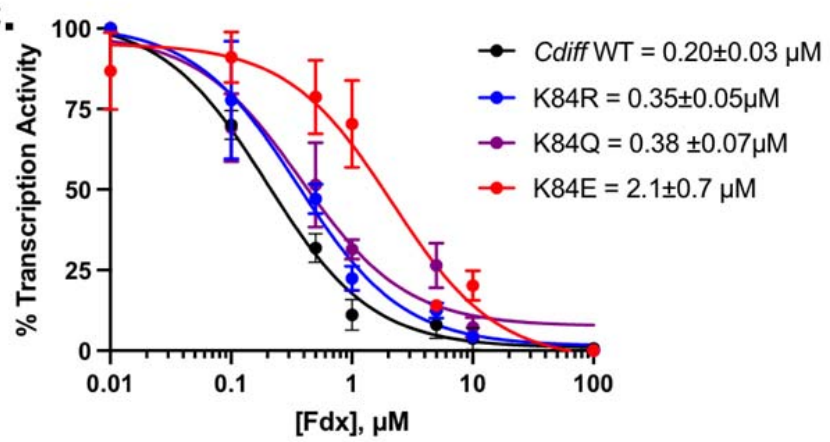

$2 \%(\mathrm{Q} / \mathrm{S} / \mathrm{T})$ $\Gamma 2 \%(K / R)$

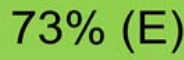

Actinobacteria

Proteobacteria b.

d.

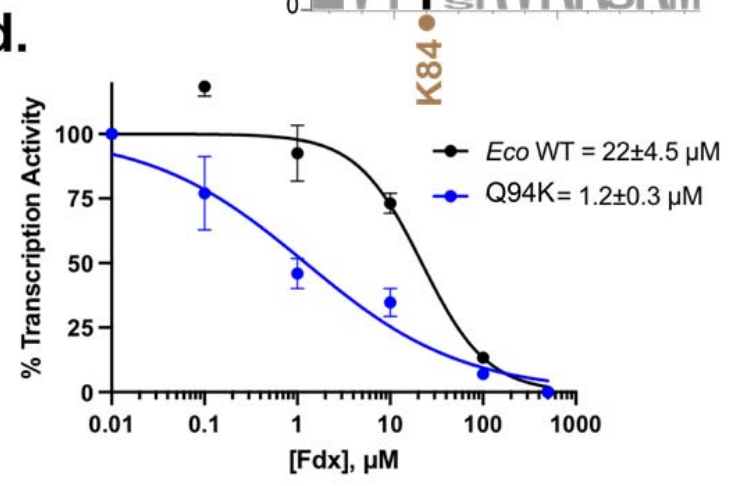

Fig. 4. The sensitizer position ( $\beta^{\prime}$ K84 in Cdiff RNAP) explains Fdx narrow-spectrum activity in the gut microbiota. a, The amino acid present at the sensitizer position among the four most common bacterial phyla in the human microbiota (specified after \%) compared to the relative abundance of bacteria in each phylum shown in the pie chart as a percent of total microbiota (adapted from Refs. ${ }^{31}$ and ${ }^{30}$ ). The amino acids present at the sensitizer position for each phylum were identified based on 66 representative species ${ }^{28}{ }^{29}$ (Extended Data Fig.10, Extended Data Table 4). $\mathbf{b}$, Sequence logos for the Fdx-interaction region of the $\beta^{\prime} Z B D$ is highly conserved among Bacteroidetes, Firmicutes, Actinobacteria, and Proteobacteria except for the sensitizer position and C-adjacent residue ( $\beta^{\prime} \mathrm{K} 84$ and S85 in Cdiff RNAP). The logos were derived using the same 66 representative species (Extended Data Table 4). c, Fdx effects on abortive transcription reveal that the $\beta^{\prime} \mathrm{K} 84 \mathrm{E}$ substitution increases resistance 10 -fold, whereas $\beta^{\prime} \mathrm{K} 84 \mathrm{Q}$ and $\beta^{\prime} \mathrm{K} 84 \mathrm{R}$ have much lesser effects. d, Transcription assays with $E c o E \sigma^{70}$ show the $\beta^{\prime} \mathrm{Q} 94 \mathrm{~K}$ substitution in the Eco RNAP reduces Fdx IC50 by a factor of $\sim 20$ relative to the WT enzyme. 
Methods

320 Structural biology software was accessed through the SBGrid consortium ${ }^{32}$.

321 Protein Expression and Purification.

322 Cdiff $\boldsymbol{\sigma}^{\mathrm{A}}$

323 The Cdiff $\sigma^{\mathrm{A}}$ gene (Kyoto Encyclopedia of Genes and Genomes (KEGG) entry: CD630_14550)

324 was amplified from Cdiff 630 chromosomal DNA and cloned between NcoI and NheI sites of 325 pET28a plasmid. A His 10 tag with a Rhinovirus $3 \mathrm{C}$ protease recognition site was added to the N326 terminus end of the $\sigma^{\mathrm{A}}$ gene to facilitate purification. Escherichia coli (Eco) BL21 (DE3) cells 327 were transformed with this plasmid and were induced with $0.5 \mathrm{mM}$ isopropyl-beta-D-

328 thiogalactopyranoside (IPTG) overnight at $16^{\circ} \mathrm{C}$. The protein was affinity purified on a $\mathrm{Ni}^{2+}-$ 329 column (HiTrap IMAC HP, GE Healthcare Life Sciences). The eluted protein was cleaved with 330 Rhinovirus 3C protease overnight and the cleaved complex was loaded onto a second $\mathrm{Ni}^{2+}$ 331 column and the flow-through collected and further purified by size exclusion chromatography 332 (Superdex 200, GE Healthcare) in buffer containing $20 \mathrm{mM}$ Tris-HCl, pH 8, 5\% (v/v) glycerol, 1 333 mM EDTA, $0.5 \mathrm{M} \mathrm{NaCl}$, and $5 \mathrm{mM}$ DTT. The eluted Cdiff $\sigma^{\mathrm{A}}$ were subsequently concentrated 334 and stored at $-80^{\circ} \mathrm{C}$.

\section{Cdiff RNAP}

336 The Cdiff RNAP overexpression plasmid was constructed in multiple steps. First, the Cdiff 630 337 rpoA (KEGG: CD630_00980), rpoZ (KEGG: CD630_25871), rpoB (KEGG: CD630_00660), 338 and rpoC (KEGG: CD630_00670) genes were codon-optimized for Eco using Gene Designer 339 (ATUM, Inc.) and codon frequencies reported by Welch et $\mathrm{al}^{33}$. A strong ribosome-binding site 340 (RBS) was designed for each gene using the Salis RBS design tools

341 (https://www.denovodna.com/software/) ${ }^{34}$. gBlock fragments containing rpoA, rpoZ, rpoB and 342 rроC genes and corresponding RBS were purchased from Integrated DNA Technologies directly. 343 DNA fragments were assembled into pET21 (Novagen) using Gibson Assembly (NEB). Next, to 344 maintain the subunit stoichiometry and prevent assembly with the host Eco subunits, $\beta$ and $\beta^{\prime}$

345 were fused using a polypeptide linker (LARHGGSGA). The same method was also used to 346 construct overexpression plasmid for $M t b$ RNAP $^{35}$. Finally, the His 10 tag with a Rhinovirus 3C 
347 protease cleavable site was added to the C-terminus of $r p o C$ to facilitate purification, resulting in 348 plasmid pXC.026. The plasmids encoding Cdiff RNAP mutants (rpoC K84E, K84R, and K84Q)

349 were constructed by Q5 site-directed mutagenesis (NEB) using pXC.026 as the template.

350 Overexpression of Cdiff RNAP yielded high levels of proteolysis and inclusion bodies in the 351 conventional BL21 $\lambda$ DE3 strain. The yields of soluble Cdiff RNAP were increased in B834

352 (DE3), a strain reported to successfully produce gram-positive Bacillus subtilis (Bsub) RNAP ${ }^{36}$,

353 The Cdiff core RNAP subunits were co-overexpressed in Eco B834 (Novagen) cells overnight at $35416^{\circ} \mathrm{C}$ for $\sim 16 \mathrm{~h}$ after induction with $0.3 \mathrm{mM}$ IPTG. The cell pellet was resuspended in the lysis 355 buffer (50 mM Tris- $\mathrm{HCl} \mathrm{pH} 8.0,1 \mathrm{mM}$ EDTA, 5\% (v/v) glycerol, $5 \mathrm{mM}$ 1,4-dithiothreitol 356 (DTT), $1 \mathrm{mM}$ protease inhibitor cocktail (PIC), and $1 \mathrm{mM}$ phenylmethylsulfonyl fluoride 357 (PMSF)). Cells were lysed by continuous flow through a French press (Avestin) and spun at $35815,000 \mathrm{rpm}$ twice for $20 \mathrm{~min}$ each. Then the supernatant was then precipitated by the addition of $3590.6 \%$ polyethyleneimine (PEI). PEI pellets were washed three times with a buffer containing 10 $360 \mathrm{mM}$ Tris-HCl, $\mathrm{pH}$ 8, $0.25 \mathrm{M} \mathrm{NaCl}, 0.1 \mathrm{mM}$ EDTA, $5 \mathrm{mM} \mathrm{DTT}$, and 5\% (v/v) glycerol, and 361 consequently eluted three times with a buffer of the same composition but with $1 \mathrm{M} \mathrm{NaCl}$.

362 Protein was precipitated overnight with $35 \%$ (w/v) ammonium sulfate and resuspended in 20 $363 \mathrm{mM}$ Tris-HCl, $\mathrm{pH} 8,5 \%$ (v/v) glycerol, $0.5 \mathrm{M} \mathrm{NaCl}$, and $5 \mathrm{mM} \beta$-mercaptoethanol. The sample 364 was then subject to $\mathrm{Ni}^{2+}$-affinity chromatography purification. The eluant protein was dialyzed 365 overnight in a buffer $20 \mathrm{mM}$ Tris- $\mathrm{HCl} \mathrm{pH} 8.0,5 \%$ (v/v) glycerol, $0.1 \mathrm{mM}$ EDTA, $0.5 \mathrm{M} \mathrm{NaCl}$, 366 and $1 \mathrm{mM}$ DTT, and subsequently concentrated and stored at $-80^{\circ} \mathrm{C}$.

\section{In vitro Transcription Assays}

368 Transcription assays were performed as described previously ${ }^{37}$. Briefly, $50 \mathrm{nM}$ of Cdiff/Eco WT 369 or mutant RNAP E $\sigma^{\mathrm{A}}$ in transcription buffer $(10 \mathrm{mM}$ Tris $\mathrm{HCl}, \mathrm{pH} 7.9,170 \mathrm{mM} \mathrm{NaCl}, 10 \mathrm{mM}$ $370 \mathrm{MgCl}_{2}, 1 \mathrm{mM}$ DTT, $5 \mu \mathrm{g} / \mathrm{ml}$ bovine serum albumin (BSA) and $0.1 \mathrm{mM}$ EDTA) was mixed with 371 different concentrations of $\mathrm{Fdx}(0.01-500 \mu \mathrm{M})$. The mixtures were incubated at $37{ }^{\circ} \mathrm{C}$ for $5 \mathrm{~min}$ 372 to allow for the antibiotic to bind. The Cdiff rrnC (GenBank: CP010905.2) dsDNA was added $373(10 \mathrm{nM})$ to each tube and the samples were incubated for an additional $15 \mathrm{~min}$ at $37^{\circ} \mathrm{C}$ to allow 374 the formation of the RNAP open complex. Then the dinucleotide $(\mathrm{GpU}, 20 \mu \mathrm{M})$ was added and 
375 incubated for $10 \mathrm{~min}$. Transcription was initiated by adding a nucleotide mixture consisting of 20

$376 \mu \mathrm{M}$ GTP and $1.25 \mu \mathrm{Ci}(15 \mathrm{nM})-\alpha-{ }^{32} \mathrm{P}-\mathrm{GTP}$. Each reaction was allowed to proceed for $10 \mathrm{~min}$ at

$37737^{\circ} \mathrm{C}$ and reactions were quenched by the addition of $2 \mathrm{X}$ stop buffer $(0.5 \mathrm{X}$ TBE, pH 8.3, $8 \mathrm{M}$

378 urea, $30 \mathrm{mM}$ EDTA, 0.05\% bromophenol blue, and 0.05\% xylene cyanol). Reactions were

379 heated at $95^{\circ} \mathrm{C}$ for $1 \mathrm{~min}$ and loaded onto a polyacrylamide gel [20\% Acrylamide/Bis acrylamide

380 (19:1), 6M urea, and 1X TBE, pH 8.3]. Transcription products were visualized by using

381 Typhoon FLA 9000 (GE Healthcare) and quantified using ImageQuant software (GE

382 Healthcare). Quantified values were plotted in PRISM and the IC50 was calculated from three

383 independent data sets. The full sequences of the fragments (initiation sites in lowercase, -35 and

$384-10$ elements in bold) used for transcription are as follows:

385 rrnC:

386 AATAGCTTGTATTAAAGCAGTTAAAATGCATTAATATAGGCTATTTTTATTTTGACA 387 AAAAAATATTTAAAATAAAAGTTAAAAAGTTGTTGACTTAGAATAATATAGATGAT 388 ATTATATATGAgtgCCCAAAAGGAGCACCAAAATAAGACAAAAGAACTTTGAAAATT 389 AAACAGTA

\section{Preparation of WT Cdiff E $\sigma^{\mathbf{A}}$ for cryo-EM}

391 The RNAP core was incubated with 15 molar excess of $\sigma^{\mathrm{A}}$ for $15 \mathrm{~min}$ at $37^{\circ} \mathrm{C}$ and $45 \mathrm{~min}$ at $3924^{\circ} \mathrm{C}$. Then, the complex was purified over a Superose 6 Increase 10/300 GL column (GE 393 Healthcare, Pittsburgh, PA) in gel filtration buffer (20 mM Tris-HCl pH 8.0, $150 \mathrm{mM} \mathrm{K-}$

394 glutamate, $5 \mathrm{mM} \mathrm{MgCl}_{2}, 2.5 \mathrm{mM}$ DTT). The eluted RNAP E $\sigma^{\mathrm{A}}$ was concentrated to $6 \mathrm{mg} / \mathrm{mL}$ $395(14 \mu \mathrm{M})$ by centrifugal filtration (Amicon Ultra). E $\sigma^{\mathrm{A}}$ was mixed with $100 \mu \mathrm{M}$ final 396 concentration of Fdx (10 mM stock solution in DMSO) and incubated for 15 minutes at $4{ }^{\circ} \mathrm{C}$.

397 Cryo-EM grid preparation

398 Before freezing cryo-EM grids, octyl $\beta$-D-glucopyranoside was added to the samples to a final 399 concentration of $0.1 \%{ }^{8}$. C-flat holey carbon grids (CF-1.2/1.3-4Au, Protochips, Morrisville, NC) 400 were glow-discharged for $20 \mathrm{sec}$ before the application of $3.5 \mu \mathrm{L}$ of the samples. Using a 401 Vitrobot Mark IV (Thermo Fisher Scientific Electron Microscopy, Hillsboro, OR), grids were 402 blotted and plunge-froze into liquid ethane with $100 \%$ chamber humidity at $22^{\circ} \mathrm{C}$. 


\section{Cryo-EM data acquisition and processing}

404 Cdiff $\sigma^{\mathrm{A}}$ with Fdx grids were imaged using a $300 \mathrm{keV}$ Titan Krios (Thermo Fisher Scientific 405 Electron Microscopy) equipped with a K3 Summit direct electron detector (Gatan, Pleasanton, 406 CA). Dose-fractionated movies were recorded in counting mode using Leginon at a nominal 407 pixel size of $1.083 \AA / p x$ (micrograph dimensions of 5,760 x 4,092 px) over a nominal defocus 408 range of $-1 \mu \mathrm{m}$ to $-2.5 \mu \mathrm{m}^{38}$. Movies were recorded in "counting mode" (native K3 camera 409 binning 2) with a dose rate of 30 electrons/physical pixel/s over a total exposure of $2 \mathrm{~s}$ (50 410 subframes of $0.04 \mathrm{~s}$ ) to give a total dose of $\sim 51 \mathrm{e}-/ \AA^{\wedge} 2$. A total of 6,930 movies were collected.

411 Dose-fractionated movies were gain-normalized, drift-corrected, summed, and dose-weighted 412 using MotionCor ${ }^{39}$. The contrast transfer function was estimated for each summed image using 413 Patch CTF module in cryoSPARC v2.15.0 ${ }^{40}$. cryoSPARC Blob Picker was used to pick particles 414 (no template was supplied). A total of 2,502,242 particles were picked and extracted from the 415 dose-weighted images in cryoSPARC using a box size of 256 pixels. Particles were sorted using cryoSPARC 2D classification (number of classes, $\mathrm{N}=50$ ), resulting in 2,415,902 curated

417 particles. Initial models (Ref 1: RNAP, Ref 2: decoy 1, Ref 3: decoy 2) were generated using 418 cryoSPARC Ab initio Reconstruction ${ }^{40}$ on a subset of 81,734 particles. Particles were further 419 curated using Ref 1-3 as 3D templates for cryoSPARC Heterogeneous Refinement $(\mathrm{N}=6)$, 420 resulting in the following: class1 (Ref 1), 464,460 particles; class2 (Ref 1), 641,091 particles; 421 class3 (Ref 2), 296,508 particles; class4 (Ref 2), 296,203 particles; class5 (Ref 3), 390,575 422 particles; class6 (Ref 3), 327,065 particles. Particles from class1 and class2 were combined and 423 further curated with another round of Heterogeneous Refinement $(\mathrm{N}=6)$, resulting in the 424 following: class1 (Ref 1), 262,185 particles; class2 (Ref 1), 394,040 particles; class3 (Ref 2), 425 110,023 particles; class4 (Ref 2), 110,743 particles; class5 (Ref 3), 104,013 particles; class6 426 (Ref 3), 124,547 particles. Curated particles from class 2 were refined using cryoSPARC Non427 uniform Refinement ${ }^{40}$ and then further processed using RELION 3.1-beta Bayesian Polishing ${ }^{41}$. 428 Per-particle CTFs were estimated for the polished particles using cryoSPARC Homogeneous 429 Refinement with global and local CTF refinement enable ${ }^{40}$. These particles were further curated 430 using cryoSPARC Heterogeneous Refinement (N=3), resulting in the following: class1 (Ref 1), 431 85,470 particles; class2 (Ref 1), 231,310 particles; class3 (Ref 1), 77,250 particles. Particles from 
432 class2 were selected for a subsequent cryoSPARC Heterogeneous Refinement $(\mathrm{N}=3)$, resulting in

433 the following: class1 (Ref 1), 19,282 particles; class2 (Ref 1), 182,390 particles; class3 (Ref 1),

43429,638 particles. Particles in class 2 were refined using cryoSPARC Non-uniform Refinement ${ }^{42}$

435 resulting in final 3D reconstruction containing 182,390 particles with nominal resolution of 3.26

436 A. Local resolution calculations were generated using blocres and blocfilt from the Bsoft

437 package $^{43}$.

438 Model building and refinement

439 A homology model for Cdiff $\mathrm{E}^{\mathrm{A}}$ was derived using SWISS-MODEL ${ }^{44}$ and PDBs: 5 VI5 $5^{20}$ for $\alpha \mathrm{I}$

440 and $\alpha \mathrm{II} ; 6 \mathrm{BZO}^{7}$ for $\beta, \beta^{\prime}$, and $\sigma^{\mathrm{A}}$; and $6 \mathrm{FLQ}^{45}$ for $\omega$. The homology model was manually fit into

441 the cryo-EM density maps using Chimera ${ }^{46}$ and rigid-body and real-space refined using Phenix ${ }^{47}$.

442 A model of Fdx was used from the previous structure PDB ID: 6BZO to place in the cryo-EM

443 map $^{7}$. Rigid body refinement for rigid domains of RNAP was performed in PHENIX. The model

444 was then manually adjusted in $\operatorname{Coot}^{48}$ and followed by all-atom and B-factor refinement with

445 Ramachandran and secondary structure restraints in PHENIX. The BBM2 modules were

446 manually built using the $E c{ }^{16}$ (PDB ID:4LK1) and Bsub structures ${ }^{17}$ (PDB ID:6ZCA) as a

447 template. The refined model was 'shaken' by introducing random shifts to the atomic coordinates

448 with RMSD of $0.163 \AA$ in phenix.pdbtools ${ }^{47}$. The shaken model was refined into half-map1 and

449 FSCs were calculated between the refined shaken model and half-map1 (FSChalf1 or work),

450 half-map2 (FSChalf2 or free, not used for refinement), and combined (full) maps using

451 phenix.mtriage ${ }^{49}$. Unmasked log files were plotted in PRISM and the FSC-0.5 was calculated for

452 the full map.

453 Reporting summary: Further information on research design is available in the Nature Research

454 Reporting Summary linked to this paper.

455 Data and materials availability: Cryo-EM maps and atomic models have been deposited in the

456 Electron Microscopy Database (EMDB accession codes 23210) and the Protein Database (PDB

457 accession codes 7L7B). Unique materials are available from the corresponding authors on

458 request. 


\section{Methods References}

Morin, A. et al. Collaboration gets the most out of software. Elife 2, e01456, doi:10.7554/eLife.01456 (2013).

33 Welch, M. et al. Design parameters to control synthetic gene expression in Escherichia coli. PLoS One 4, e7002, doi:10.1371/journal.pone.0007002 (2009).

34 Salis, H. M., Mirsky, E. A. \& Voigt, C. A. Automated design of synthetic ribosome binding sites to control protein expression. Nat Biotechnol 27, 946-950, doi:10.1038/nbt.1568 (2009).

35 Czyz, A., Mooney, R. A., Iaconi, A. \& Landick, R. Mycobacterial RNA polymerase requires a U-tract at intrinsic terminators and is aided by NusG at suboptimal terminators. Mbio 5, e00931, doi:10.1128/mBio.00931-14 (2014).

36 Yang, X. \& Lewis, P. J. Overproduction and purification of recombinant Bacillus subtilis RNA polymerase. Protein Expr Purif 59, 86-93, doi:10.1016/j.pep.2008.01.006 (2008).

37 Davis, E., Chen, J., Leon, K., Darst, S. A. \& Campbell, E. A. Mycobacterial RNA polymerase forms unstable open promoter complexes that are stabilized by CarD. Nucleic Acids Res 43, 433-445, doi:10.1093/nar/gku1231 (2015).

38 Suloway, C. et al. Automated molecular microscopy: the new Leginon system. J Struct Biol 151, 41-60, doi:10.1016/j.jsb.2005.03.010 (2005).

39 Zheng, S. Q. et al. MotionCor2: anisotropic correction of beam-induced motion for improved cryo-electron microscopy. Nat Methods 14, 331-332, doi:10.1038/nmeth.4193 (2017).

40 Punjani, A., Rubinstein, J. L., Fleet, D. J. \& Brubaker, M. A. cryoSPARC: algorithms for rapid unsupervised cryo-EM structure determination. Nat Methods 14, 290-296, doi:10.1038/nmeth.4169 (2017).

41 Zivanov, J. et al. New tools for automated high-resolution cryo-EM structure determination in RELION-3. Elife 7, doi:10.7554/eLife.42166 (2018).

42 Punjani, A., Zhang, H. \& Fleet, D. J. Non-uniform refinement: adaptive regularization improves singleparticle cryo-EM reconstruction. Nat Methods 17, 1214-1221, doi:10.1038/s41592-020-00990-8 (2020).

43 Heymann, J. B. \& Belnap, D. M. Bsoft: image processing and molecular modeling for electron microscopy. J Struct Biol 157, 3-18, doi:10.1016/j.jsb.2006.06.006 (2007).

44 Waterhouse, A. et al. SWISS-MODEL: homology modelling of protein structures and complexes. Nucleic Acids Res 46, W296-W303, doi:10.1093/nar/gky427 (2018).

45 Guo, X. et al. Structural Basis for NusA Stabilized Transcriptional Pausing. Mol Cell 69, 816-827 e814, doi:10.1016/j.molcel.2018.02.008 (2018).

46 Pettersen, E. F. et al. UCSF Chimera--a visualization system for exploratory research and analysis. $J$ Comput Chem 25, 1605-1612, doi:10.1002/jcc.20084 (2004).

47 Afonine, P. V. et al. Real-space refinement in PHENIX for cryo-EM and crystallography. Acta Crystallogr D Struct Biol 74, 531-544, doi:10.1107/S2059798318006551 (2018).

48 Emsley, P., Lohkamp, B., Scott, W. G. \& Cowtan, K. Features and development of Coot. Acta Crystallogr D Biol Crystallogr 66, 486-501, doi:10.1107/S0907444910007493 (2010).

49 Afonine, P. V. et al. New tools for the analysis and validation of cryo-EM maps and atomic models. Acta Crystallogr D Struct Biol 74, 814-840, doi:10.1107/S2059798318009324 (2018).

50 Hubin, E. A. et al. Structural, functional, and genetic analyses of the actinobacterial transcription factor RbpA. Proc Natl Acad Sci U S A 112, 7171-7176, doi:10.1073/pnas.1504942112 (2015).

51 Wallace, A. C., Laskowski, R. A. \& Thornton, J. M. LIGPLOT: a program to generate schematic diagrams of protein-ligand interactions. Protein Eng 8, 127-134, doi:10.1093/protein/8.2.127 (1995).

52 Stamatakis, A. RAxML-VI-HPC: maximum likelihood-based phylogenetic analyses with thousands of taxa and mixed models. Bioinformatics 22, 2688-2690, doi:10.1093/bioinformatics/btl446 (2006).

53 Letunic, I. \& Bork, P. Interactive Tree Of Life (iTOL) v4: recent updates and new developments. Nucleic Acids Res 47, W256-W259, doi:10.1093/nar/gkz239 (2019).

Acknowledgments: We thank Seth Darst for helpful discussions during this study. We thank Rachel Mooney for providing $\sigma^{70}$ protein for transcription assays. We thank M. Ebrahim, J. Sotiris, and Honkit Ng at The Rockefeller University Evelyn Gruss Lipper Cryo-electron 
511 Microscopy Resource Center. Some of this work was performed at the Simons Electron

512 Microscopy Center and National Resource for Automated Molecular Microscopy located at the

513 New York Structural Biology Center, supported by grants from the Simons Foundation

514 (SF349247), NYSTAR, the Agouron Institute (F00316), and the NIH (GM103310, OD019994).

515 We thank Ed Eng and Kashyap Maruthi for collecting cryo-EM data. This research was

516 supported by grants from the NIH to R.L. (GM38660) and E.A.C. (GM114450).

517 Author contributions:

518 E.A.C. and R.L. supervised this work. X.C. and H.B. carried out biochemical and functional

519 assays. H.B. and J.C. determined the cryo-EM structures. X.C. performed bioinformatic analysis.

520 Y.B. assisted with protein purifications. X.C., H.B., E.A.C., and R.L. wrote the manuscript with

521 input from all authors.

522 Competing interests: Authors declare that they have no competing interests.

523 Additional information

524 Supplementary Information is available for this paper.

525 Correspondence and requests for materials should be addressed to Elizabeth A. Campbell and 526 Robert Landick. 

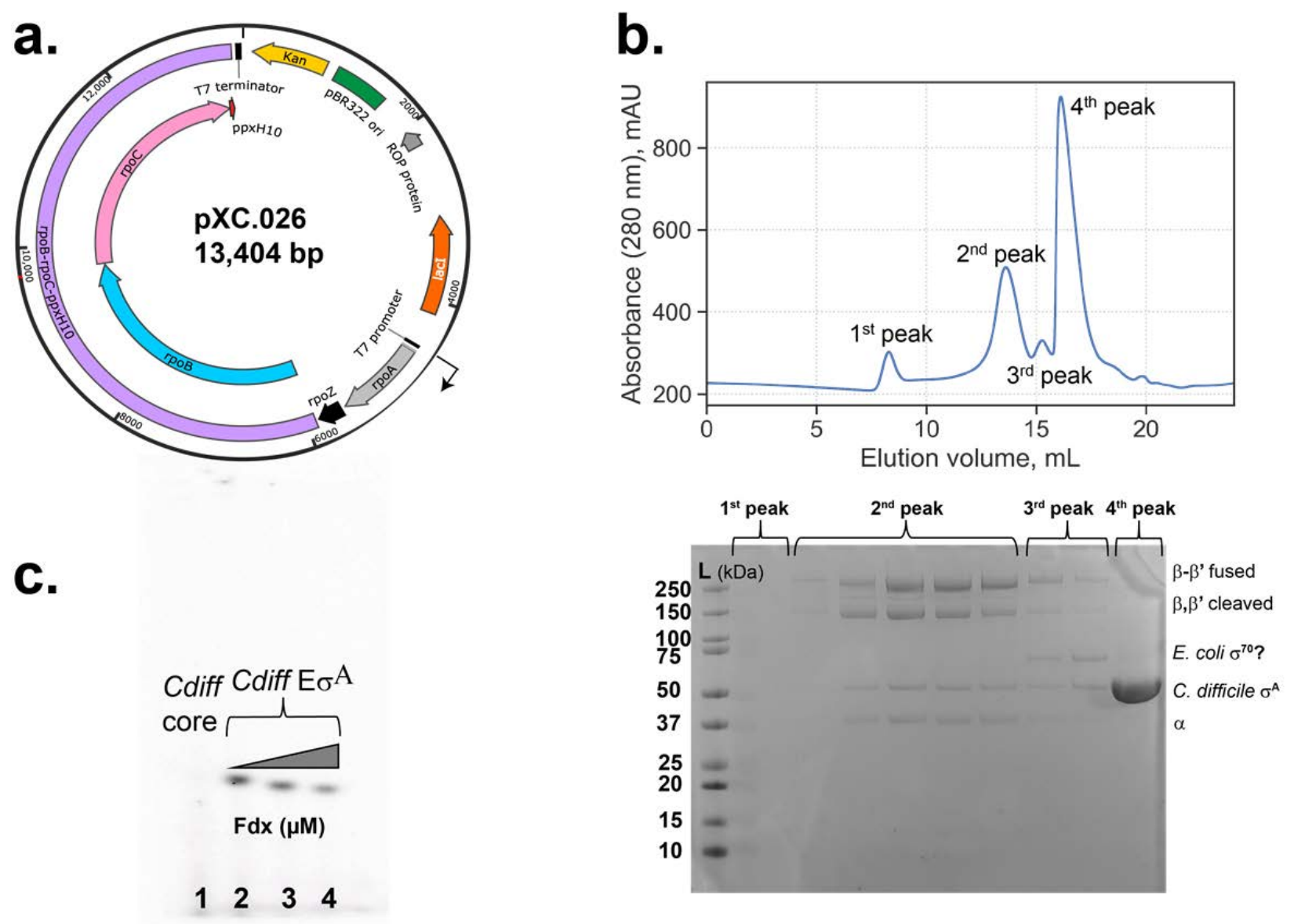

543 Extended Data Fig. 1. Overexpression and purification of Cdiff RNAP. a, pXC.026,

544 overexpression plasmid for the Cdiff rpoA, rpoZ, rpoB, and $r p o C$ genes (encoding the $\alpha, \omega, \beta$,

545 and $\beta^{\prime}$ subunits of Cdiff RNAP, respectively). The $\beta$ and $\beta^{\prime}$ subunits were fused with an inter-

546 subunit 10-amino-acid (aa) linker (LARHVGGSGA) and a C-terminal Rhinovirus 3C protease-

547 cleavable $\mathrm{His}_{10}$ tag. b, (Top) Size-exclusion chromatography profile for the assembled Cdiff

548 RNAP E $\sigma^{\mathrm{A}}$. (Bottom) Coomassie-stained SDS-PAGE of individual fractions from major peaks.

549 RNAP subunits are labeled on the right of the gel. Cdiff RNAP for biochemistry and structural

550 biology was taken from pooled fractions of the second peak. c, Abortive transcription assay with

551 Cdiff core and $\mathrm{E}^{\mathrm{A}}$ using the Cdiff $r r n C$ promoter as DNA template. The transcriptional activity

552 of Cdiff $\sigma^{\mathrm{A}}$ was inhibited with increasing concentrations of Fdx. Lane 1, Cdiff RNAP core; lane

5532 , Cdiff $\mathrm{E} \sigma^{\mathrm{A}}$; lane 3, Cdiff E $\sigma^{\mathrm{A}}$ with $0.2 \mu \mathrm{M}$ Fdx added; lane 4, Cdiff $\mathrm{E} \sigma^{\mathrm{A}}$ with $2 \mu \mathrm{M}$ Fdx added. 

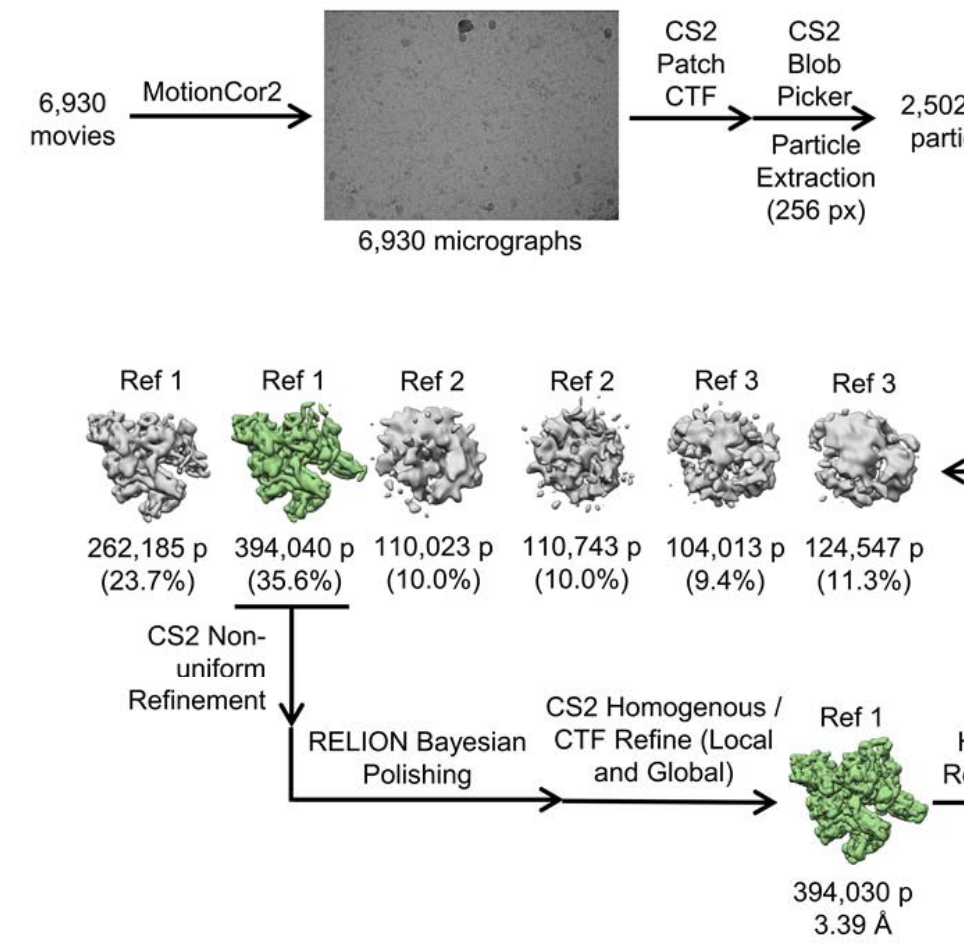

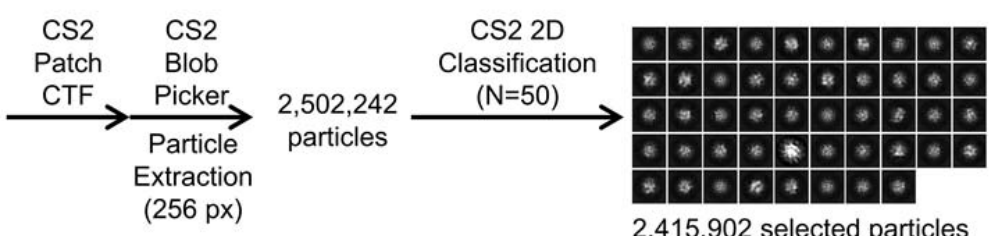

CS2 Ab initio Reconstruction (81,734 sub-selected particles, $\mathrm{N}=3$ )

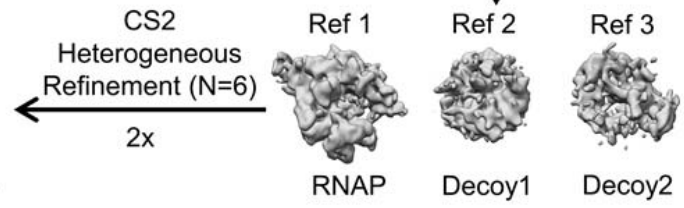

Extended Data Fig. 2. Cryo-EM processing pipeline. Flow chart showing the imageprocessing pipeline for the cryo-EM data of $C \operatorname{diff} \mathrm{E}^{\mathrm{A}} / \mathrm{Fdx}$ complexes, starting with 6,930 dosefractionated movies collected on a 300-keV Titan Krios (FEI) equipped with a K3 Summit direct electron detector (Gatan). Movies were frame-aligned and summed using MotionCor $2^{39}$. CTF estimation for each micrograph was calculated with cryoSPARC ${ }^{40}$. A representative micrograph is shown following processing by MotionCor ${ }^{39}$. Particles were auto-picked from each micrograph with cryoSPARC2 ${ }^{40}$ Blob Picker and then sorted by 2D classification using cryoSPARC2 to assess quality. The selected classes from the 2D classification are shown. After picking and cleaning by 2D, the dataset contained 2,415,902 particles. A subset of particles was used to generate an $a b$ initio templates in cryoSPARC2 and 3D heterogeneous refinement was performed with these templates using cryoSPARC ${ }^{40}$. One major, high-resolution class emerged, which was polished using RELION ${ }^{41}$ and further cleaned with two more $3 \mathrm{D}$ heterogenous refinements. The final 182,390 particles were refined using cryoSPARC Non-Uniform refinement ${ }^{42}$. 
a.
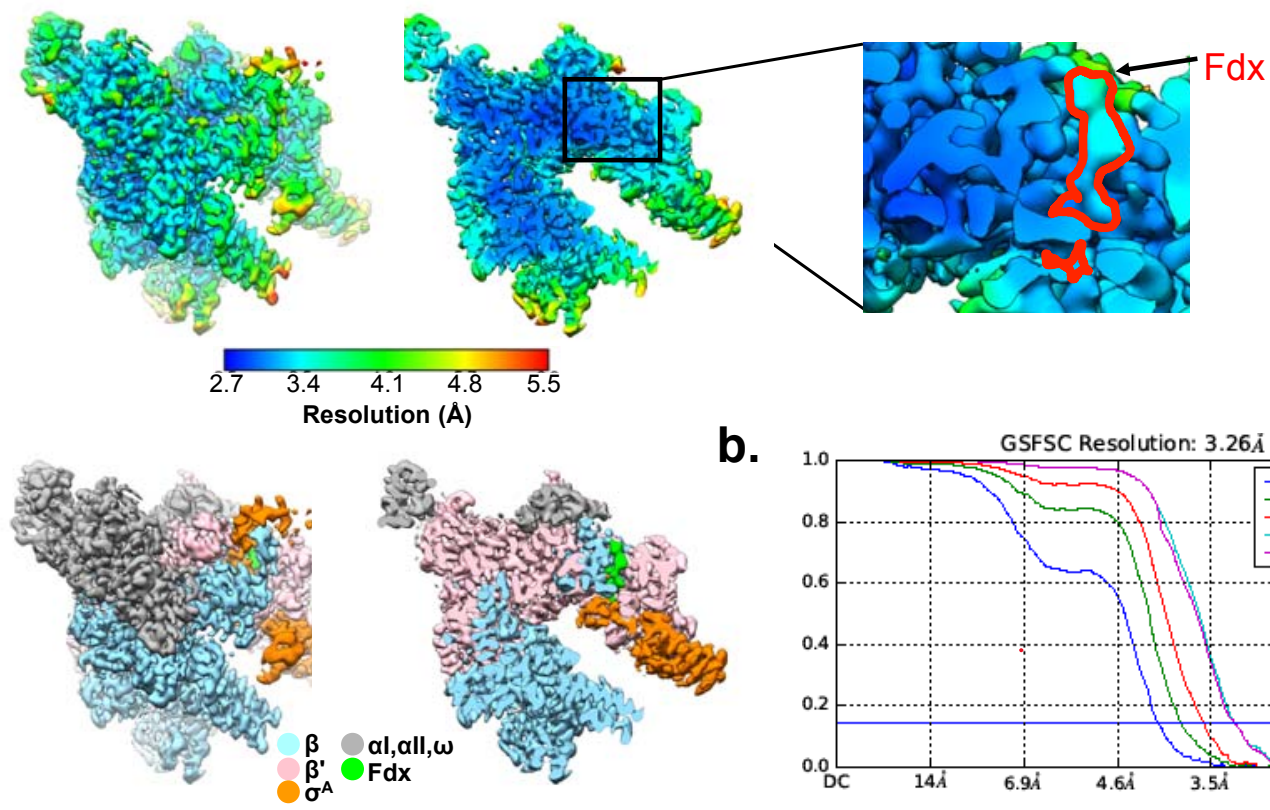

b.

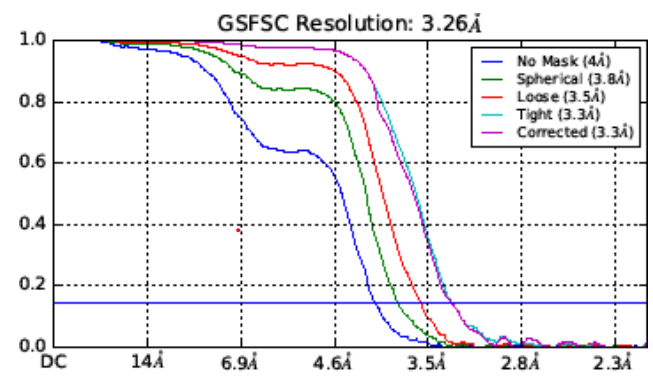

C.

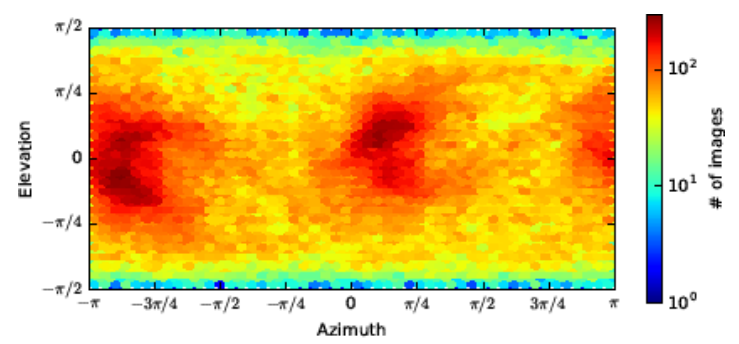

d.

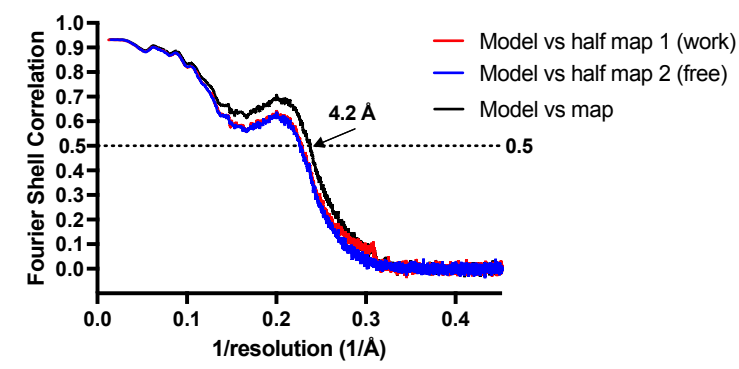

Extended Data Fig. 3. Cryo-EM analysis. a, Top left, the $3.26 \AA$-resolution cryo-EM density map of Cdiff $\mathrm{E} \sigma^{\mathrm{A}} / \mathrm{Fdx}$. Top right, a cross-section of the structure, showing the Fdx. Bottom, same views as above, but colored by local resolution, The boxed region is magnified and displayed as an inset. Density for Fdx is outlined in red. ${ }^{15} . \mathbf{b}$, Gold-standard FSC plots of the Cdiff E $\sigma^{\mathrm{A}} / \mathrm{Fdx}$ complex from cryoSPARC ${ }^{40}$. The dotted line represents the gold-standard 0.143 FSC cutoff which indicates a nominal resolution of $3.26 \AA$. c, Angular distribution calculated in cryoSPARC for $C$ diff $\mathrm{E}^{\mathrm{A}} / \mathrm{Fdx}$ particle projections. Heat map shows number of particles for each viewing angle $(\text { less }=\text { blue, more }=\text { red })^{40}$. $\mathbf{d}$, Cross-validation FSC plots for map-to-model fitting were calculated between the refined structure of $C \operatorname{diff} \mathrm{E}^{\mathrm{A}} / \mathrm{Fdx}$ and the half-map used for refinement (work, red), the other half-map (free, blue), and the full map (black). The dotted black line represents the 0.5 FSC cutoff determined for the full map ${ }^{49}$. 


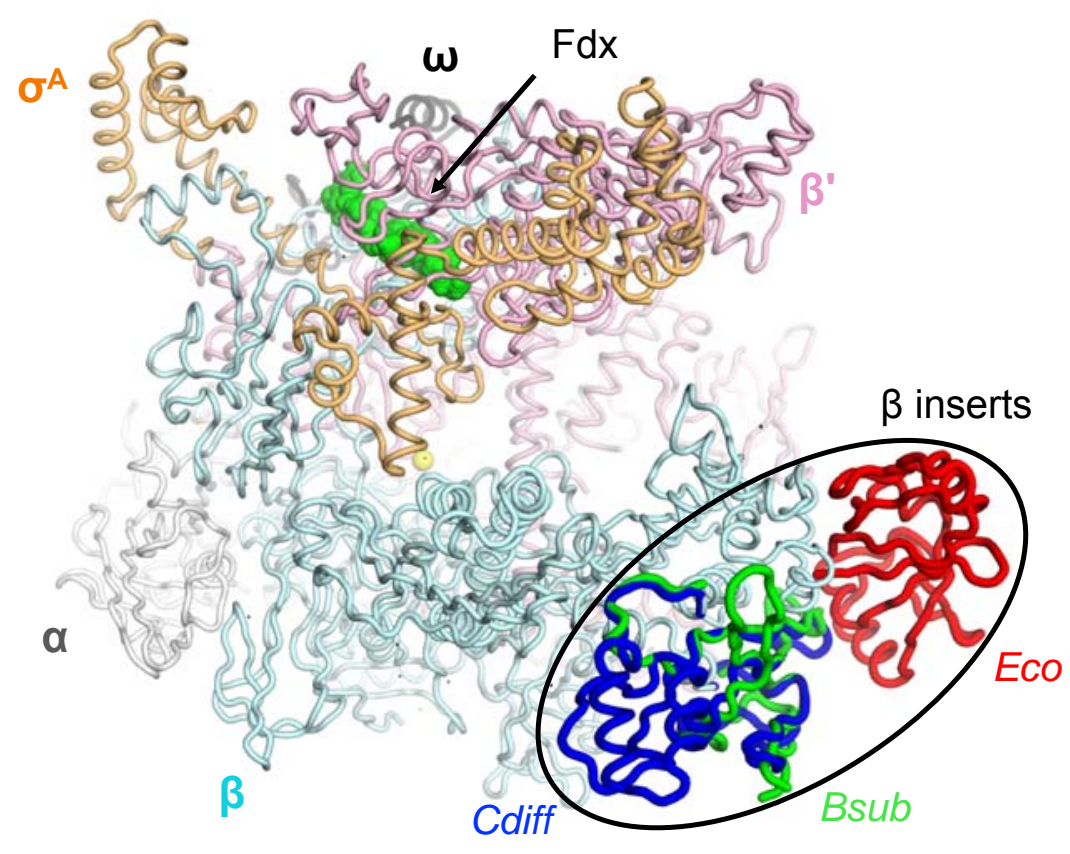

Extended Data Fig. 4. Differences between Cdiff and other bacterial RNAPs. The lineagespecific $\beta$ inserts are shown for Cdiff RNAP in dark blue, Eco RNAP in red ${ }^{16}$ (PDB ID:4LK1), Bsub RNAP in green ${ }^{17}$ (PDB ID:6ZCA). The Fdx is shown in green spheres, and the active site $\mathrm{Mg}^{2+}$ is shown as a yellow sphere. Superimposition of the RNAPs from each organism was performed in PyMOL. Only the Cdiff $\mathrm{E}^{\mathrm{A}}$ is shown. 
bioRxiv preprint doi: https://doi.org/10.1101/2022.01.17.476619; this version posted January 17,2022 . The copyright holder for this preprint (which was not certified by peer review) is the author/funder, who has granted bioRxiv a license to display the preprint in perpetuity. It is made available under aCC-BY-ND 4.0 International license.

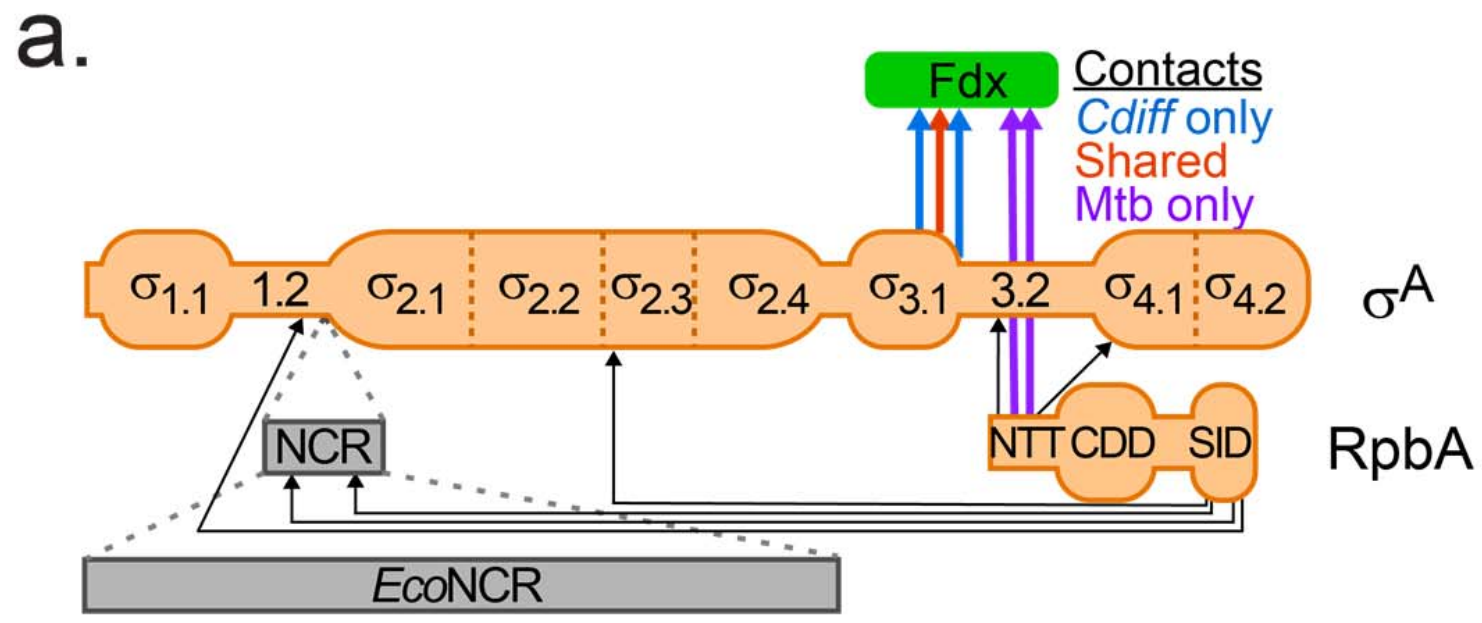

b.

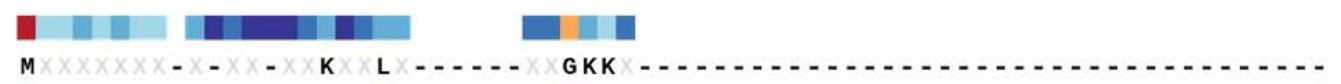

Cdf MENKSNKK-ELKK-VTAKTLI- - - - EKGKKQ - $-\ldots$

BSU MADKQTHETELTFDQVKEQLT - . $\ldots$ ESGKKR - $\ldots \ldots \ldots$

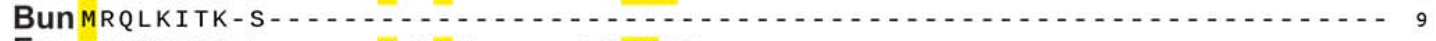

ECO MEQNPQSQ - L - . - K- LLV - . - TRGKEQ - . $\ldots \ldots$

Mtb MAATKAST - A - TDEPVKRTATKSPAASASGAKTGAKRTAAKSASGSPPAKRATKPAARSVKPASAPQDTT 68
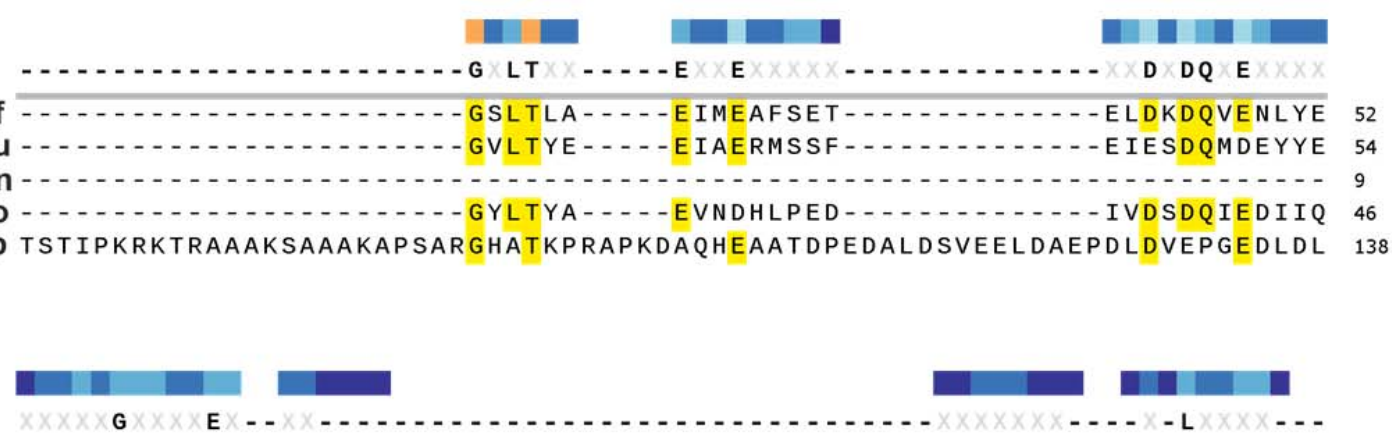

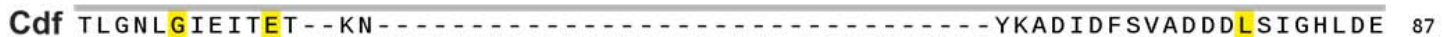

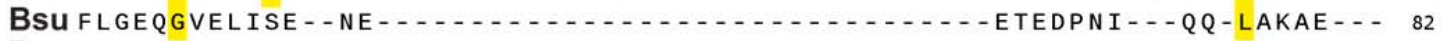

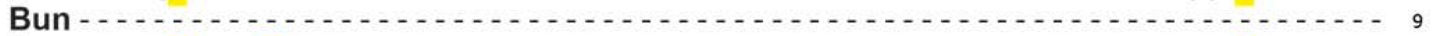

ECO MINDMG IQVMEE - - APDADD $\ldots \ldots$ Mtb DAADLNLDDLEDDVAPDADDDLDSGDDEDHEDLEAEAAVAPGQTADDDEEIAEPTEK - - - DK - ASGD - - 201

\section{- $X X X X X$ D XXXXXX}

Cdf DAEAISHDDSSAIEI - - ETVDLSLPKGISIDDPVRMYLKEIGKIPLLKPHEEVEFARRMHEGDE - - - - 149 BSU - - EEF-- - - - - DLNDLSVPPGVKINDPVRMYLKEIGRVNLLSAKEEIAYAQKIEEGDE- - - - 132 Bun - - - - - - - - - - IT - NRESASLDKYLQEIGREDLITVEEEVELAQRIRKGDR - - - - 48 ECO - - - TADEDAAEAAAQVLS SVESEI - - - GRT TDPVRMYMREMG TVELLTREGE IDIAKRIEDGINQVQCSV 134 Mtb - - FVWDEDESEALRQ - - ARKDAEL - - - TASADSVRAYLKQIGKVALLNAEEEVELAKRIEAGLY - - - - 258 
bioRxiv preprint doi: https://doi.org/10.1101/2022.01.17.476619; this version posted January 17,2022 . The copyright holder for this preprint (which was not certified by peer review) is the author/funder, who has granted bioRxiv a license to display the preprint in perpetuity. It is made available under aCC-BY-ND 4.0 International license.

\section{b. (continued)}

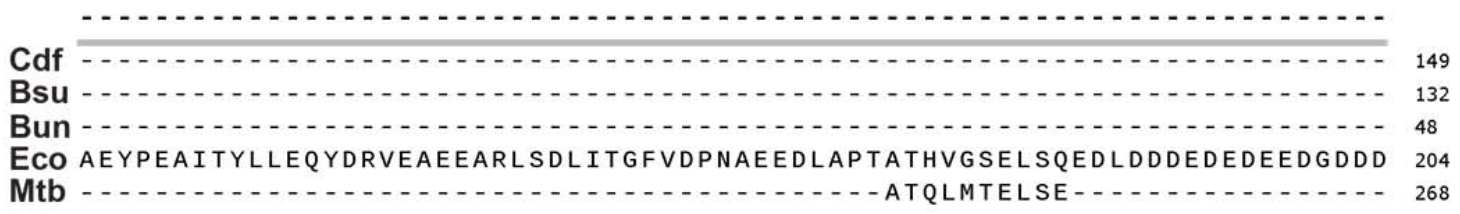

Cdf

Bsu Bun -

ECO SADDDNSIDPELAREKFAELRAQYVVTRDTIKAKGRSHATAQEEILKLSEVFKQFRLVPKQFDYLVNSMR 274 Mtb - $\ldots \ldots \ldots$
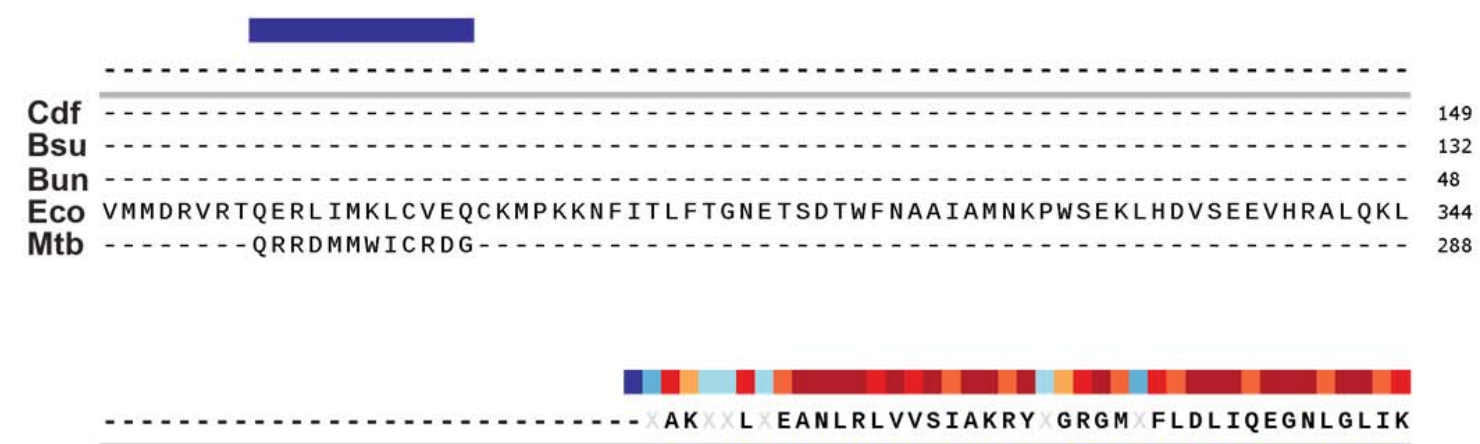

Cdf

BSu - - - - - - - - -

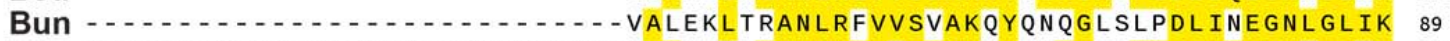

ECO QQIEEETGLTIEQVKDINRRMSIGEAKARRAKKEMVEANLRLVISIAKKYTNRGLQFLDLIQEGNIGLMK 414 Mtb -.....................................

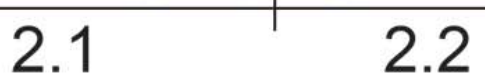

AVEKFDYTKGYKFSTYATWWIRQAITRAIADQARTIRIPVHMVETINKL R RQLLQELGREPTPEELA

Cdf AVEKFDYTKGYKFSTYATWWIRQAITRAIADQARTIRIPVHMVETINKLIRVSRQLLQELGRDPKPEEIA 260 BSU AVEKFDYRKGYKFSTYATWWIRQAITRAIADQARTIRIPVHMVETINKLIRVQRQLLQDLGREPTPEEIA 243 Bun AAEKFDETRGFKFISYAVWWIRQSILQALAEQSRIVRLPLNQVGSLNKISKAFSKFEQENERRPSPEELA 159 ECO AVDKFEYRRGYKFSTYATWWIRQA ITRSIADQARTIRIPVHMIETINKLNRISRQMLQEMGREPTPEELA 484 Mtb AVEKFDYTKGYKFSTYATWWIRQAITRAMADQARTIRIPVHMVEVINKLGRIQRELLQDLGREPTPEELA 400

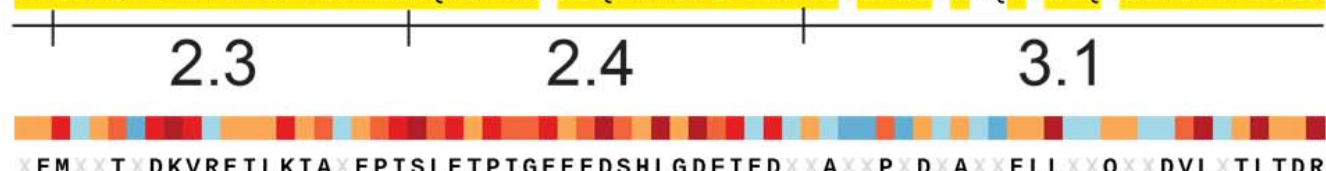

Cdf KEMEMTEDKVREIMKIAQDPVSLETPIGEEEDSHLGDFIPDDDAPAPAEAAAYSLLKEQIEDVLGSLNDR 330 BSU EDMDLTPEKVREILKIAQEPVSLETPIGEEDDSHLGDFIEDQEATSPSDHAAYELLKEQLEDVLDTLTDR 313 Bun DELEIPVDKISDTLKVSGRHISVDAPFVEGEDNSLLDVLVNDDSPMADRSLVNESLAREIDRALSTLTDR 229 ECO ERMLMPEDKIRKVLKIAKEPISMETPIGDDEDSHLGDFIEDTTLELPLDSATTESLRAATHDVLAGLTAR 554 Mtb KEMDITPEKVLEIQQYAREPISLDQTIGDEGDSQLGDFIEDSEAVVAVDAVSFTLLQDQLQSVLDTLSER 470

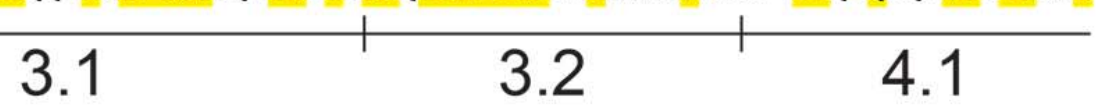




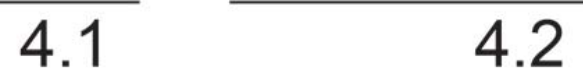
has a much shorter $\sigma^{\mathrm{A}} \mathrm{NCR}$ than $E c o \sigma^{70}$, but the residues in the short Mtb NCR that contact RbpA are not present in either Cdiff or Eco ${ }^{50}$. Mtb RbpA contacts Fdx whereas $C$ diff $\sigma^{\mathrm{A}}$ makes more contacts to $\mathrm{Fdx}$ than does $M t b \sigma^{\mathrm{A}}$. Black arrows indicate RpbA- $\sigma^{\mathrm{A}}$ contacts whereas colored arrows indicate $\mathrm{Fdx}$ contacts to $\sigma^{\mathrm{A}}$ and $\mathrm{RpbA}$, which includes one shared contact between $M t b$ and $C \operatorname{diff} \sigma^{\mathrm{A}}$ (red arrow). $\mathbf{b}$, Amino acid-sequence alignment of $\sigma^{\mathrm{A}}$ for diverse representatives of bacteria species. Identical residues are highlighted in yellow. Gaps are indicated by dashed lines. Conserved $\sigma$ regions are labeled underneath the alignment. The three letter species code is as follows: Cdf, Clostridioides difficile; Bsu, Bacillus subtilis; Bun, Bacteroides uniformis; Eco, Escherichia coli; Mtb, Mycobacterium tuberculosis. 


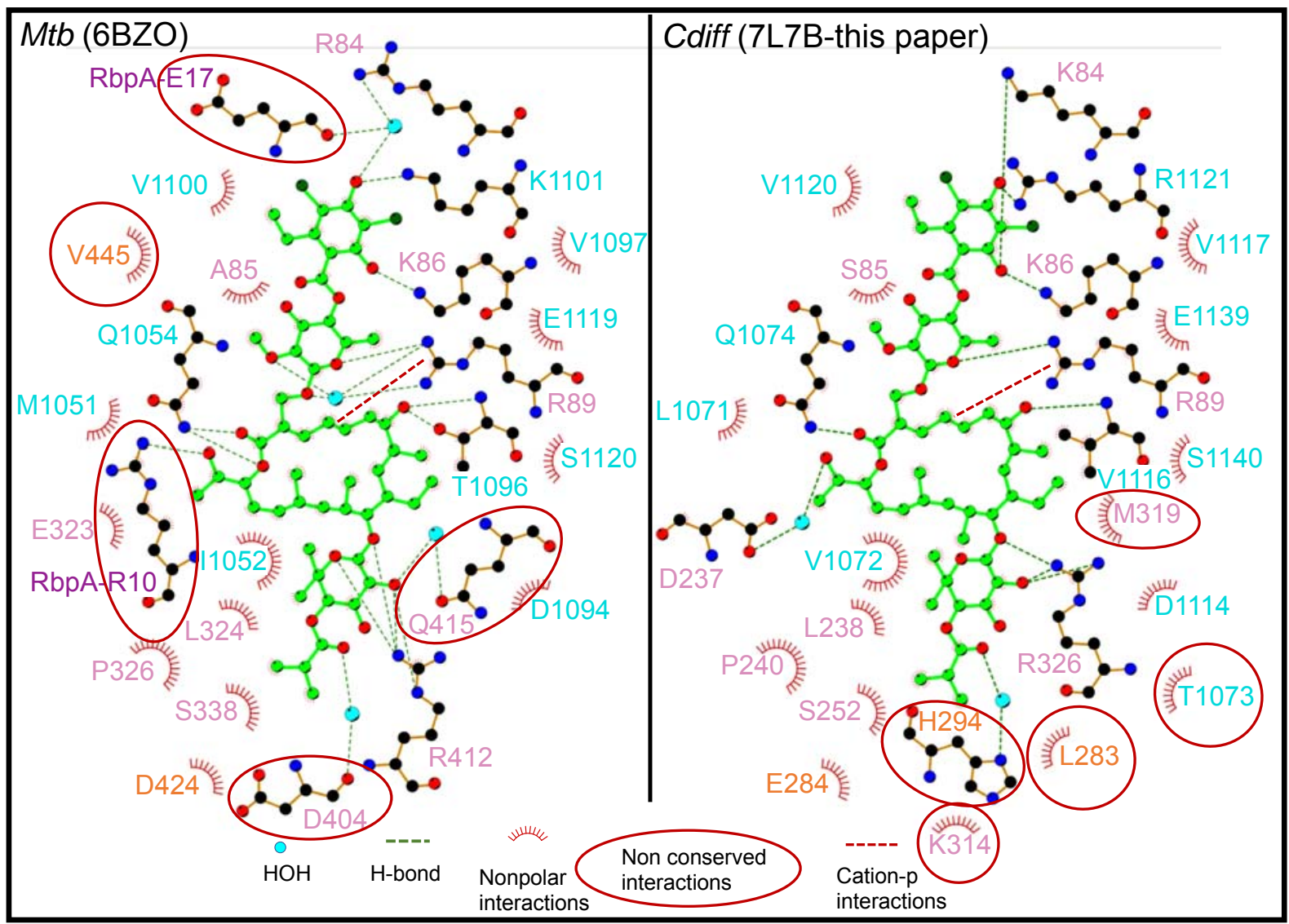

Extended Data Fig. 6 Fdx binding residues in Mtb RbpA-E $\sigma^{\mathrm{A}}$ and Cdiff E $\sigma^{\mathrm{A}}$. Ligplot $^{51}$ was used to determine contacts between Fdx and Mtb RbpA-E $\sigma^{\mathrm{A}}$ (left) and Cdiff E $\boldsymbol{\sigma}^{\mathbf{A}}$ (right). Cyan sphere, $\mathrm{H}_{2} \mathrm{O}$; green dashed line, hydrogen bond or salt bridge; red arc, van der Waals interactions; red dashed line, cation- $\pi$ interactions. Note that in ligplot of the $\operatorname{Cdiff} \mathrm{E}^{\mathrm{A}} / \mathrm{Fdx}$ interactions, V1143 (discussed in the text as one of the residues when mutated cause Fdxresistance) did not make the distance cutoff $(4.5 \AA)$ as it was located $4.7 \AA$ away from Fdx. The RNAP $\beta, \beta^{\prime}$ and $\sigma^{\mathrm{A}}$ residues are in cyan, pink, and orange respectively. The two $M t b \mathrm{RbpA}$ residues $(\mathrm{E} 17, \mathrm{R} 10)$ that interact with Fdx are colored in purple and indicated in the text. The Fdx-interacting residues that do not have corresponding interactions between Cdiff and Mtb are highlighted in red circles. 

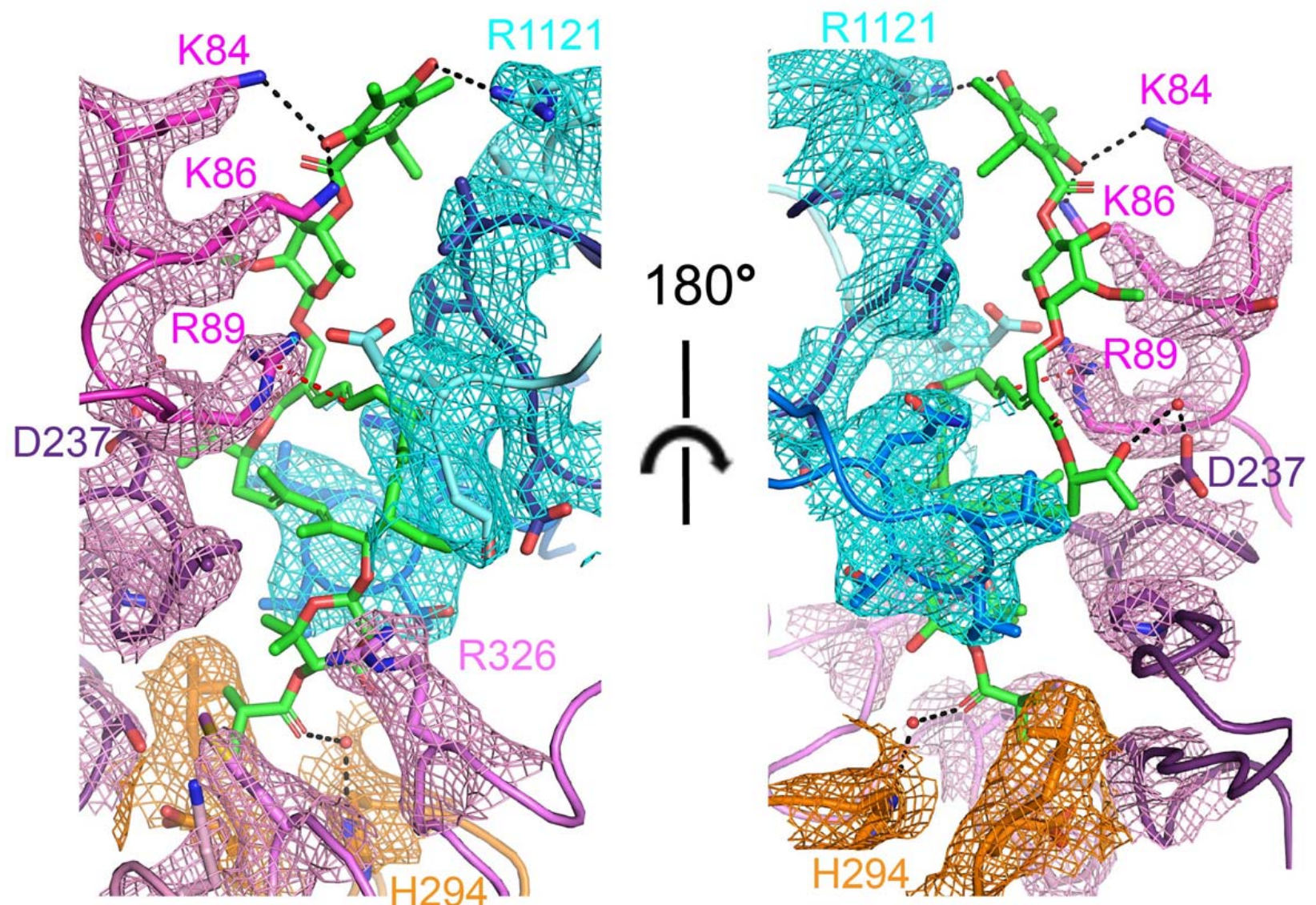

Extended Data Fig. 7. The cryo-EM density map of residues interacting with Fdx. Coloring of the residues is consistent with RNAP subunits coloring in Fig. 3, the stick model and cryo-EM densities are color-coded as follows: Pink: $\beta$-subunit, cyan: $\beta$ '- subunit, and orange: $\sigma^{\mathrm{A}}$. Water molecules are shown as red spheres. The residues that form hydrogen bonds (black dotted line) 

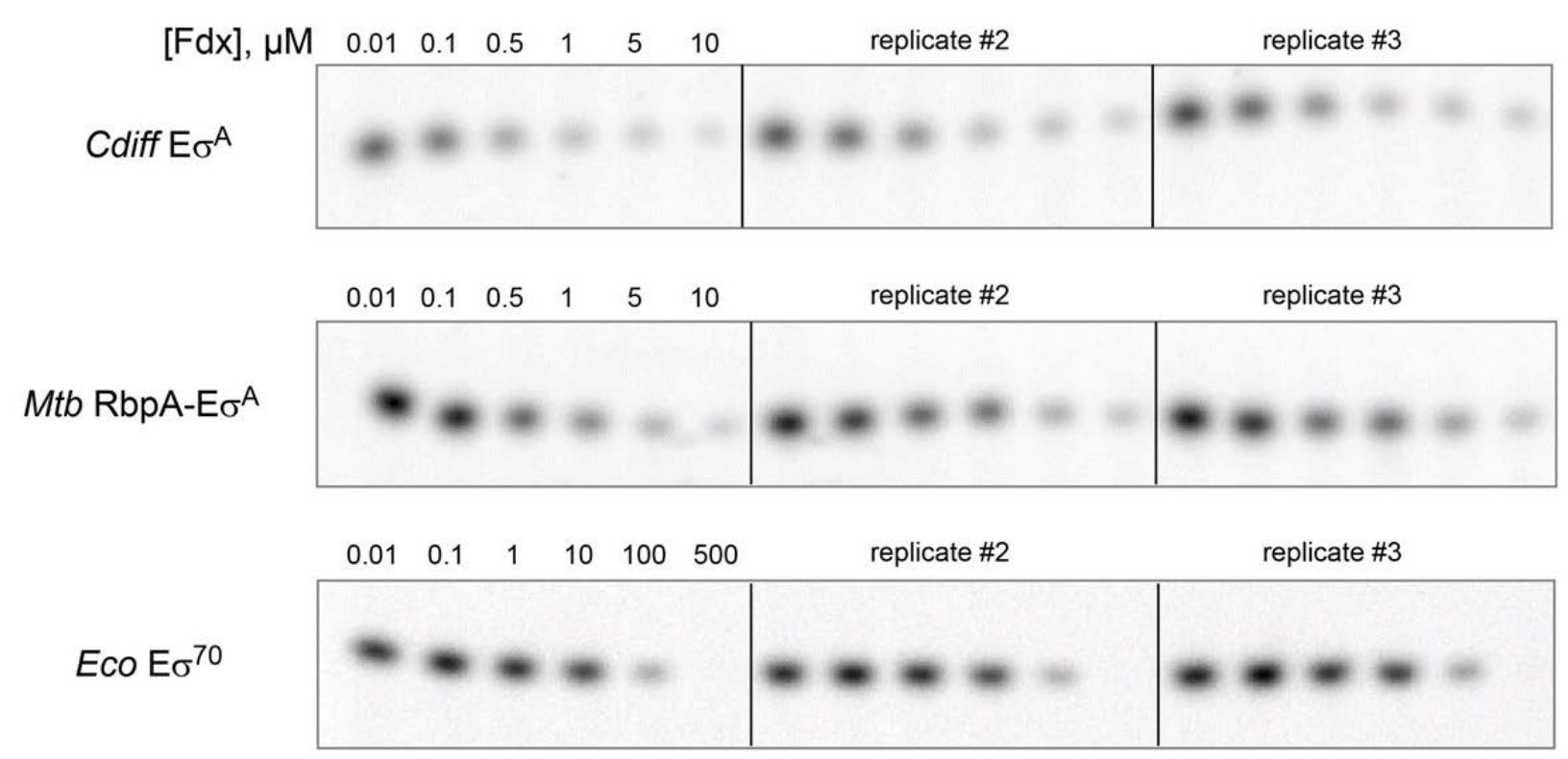

628 Extended Data Fig. 8. In vitro abortive transcription assays used to determine Fdx IC50 of $629 \boldsymbol{C d i f f}$ and $\boldsymbol{M t b} \mathbf{E} \boldsymbol{\sigma}^{\mathbf{A}}$ and $\mathbf{E} \boldsymbol{c o E} \boldsymbol{\sigma}^{\mathbf{7 0}}$. Data is plotted in Fig. 2D. Abortive ${ }^{32} \mathrm{P}-\mathrm{RNA}$ products $630 \quad\left(\mathrm{G}_{\mathrm{p}} \mathrm{U}_{\mathrm{p}} \mathrm{G}\right)$ synthesized on Cdiff rrnC promoter were quantified in the presence of increasing 631 concentrations of $\mathrm{Fdx}$. For each $\mathrm{E} \sigma^{\mathrm{A}}\left(\right.$ or $\mathrm{E} \sigma^{70}$ ), three independent experiments were performed 632 and analyzed on the same gel. 

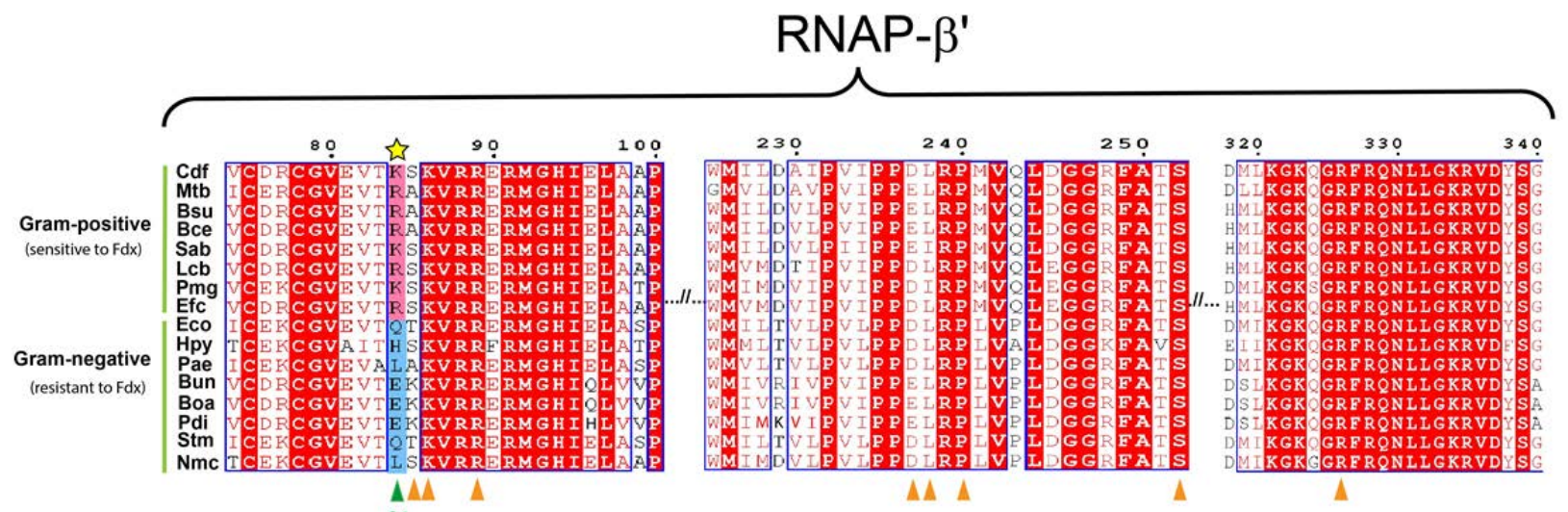

84
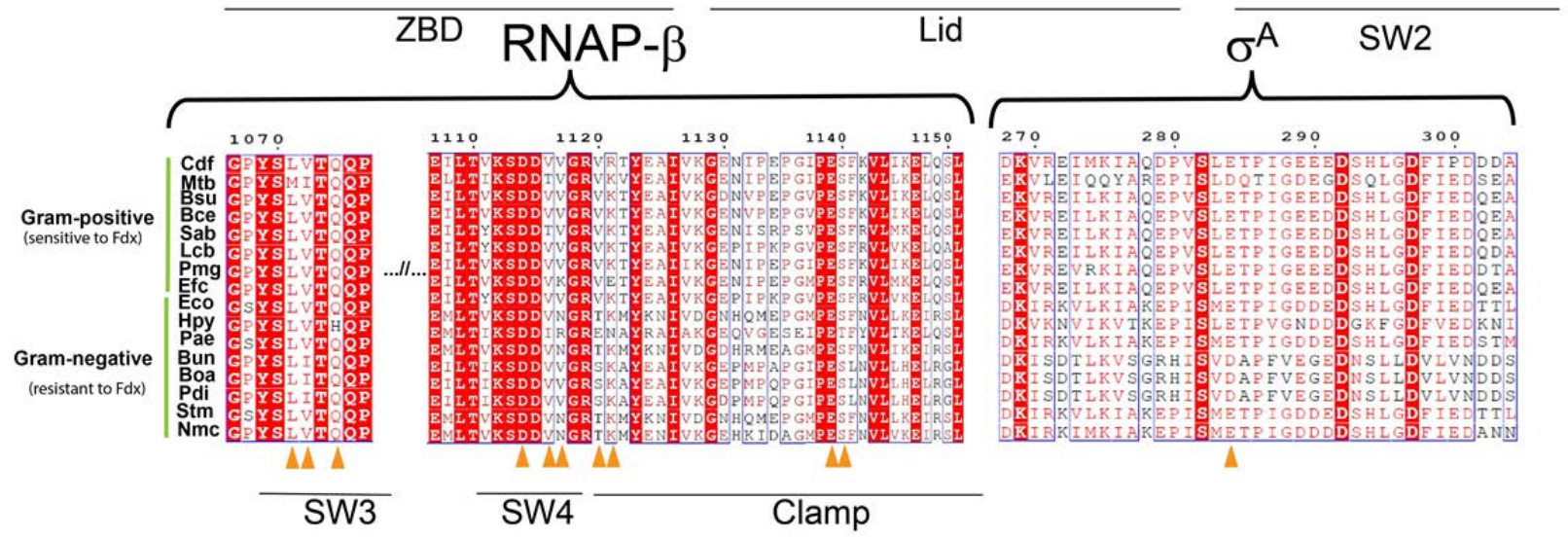

Extended Data Fig. 9. Comparative sequence alignment of key structural components of RNAPs that interact with Fdx between Fdx-resistant and sensitive bacteria. The Fdx interacting regions are labeled on the top of sequence alignment. Locations of residues contacting Fdx in both Cdiff and Mtb are labeled by triangles underneath sequences. For grampositive bacteria that are sensitive to $\mathrm{Fdx}$, the corresponding residue at Cdiff $\beta^{\prime} \mathrm{K} 84$ is either $\mathrm{K}$ or $640 \mathrm{R}$, which is highlighted in pink background. For gram-negative bacteria that are resistant to Fdx, 641 the residue at $\beta^{\prime} \mathrm{K} 84$ is neutral $\mathrm{Q}, \mathrm{L}$, or negative $\mathrm{E}$, which is highlighted in blue background. 642 Conserved residues are shown as white letters on a red background, and similar residues are 643 shown as red letters in blue boxes. Cdf, Clostridioides difficile; Mtb, Mycobacterium 644 tuberculosis; Bsu, Bacillus subtilis; Bce, Bacillus cereus, Sab, Staphylococcus aureus; Lcb, Lactobacillus casei; Pmg, Peptococcus magna; Efc, Enterococcus faecium; Eco, Escherichia coli; Hpy, Helicobacter pylori; Pae, Pseudomonas aeruginosa; Bun, Bacteroides uniformis; Boa, 648 Neisseria meningitidis. 


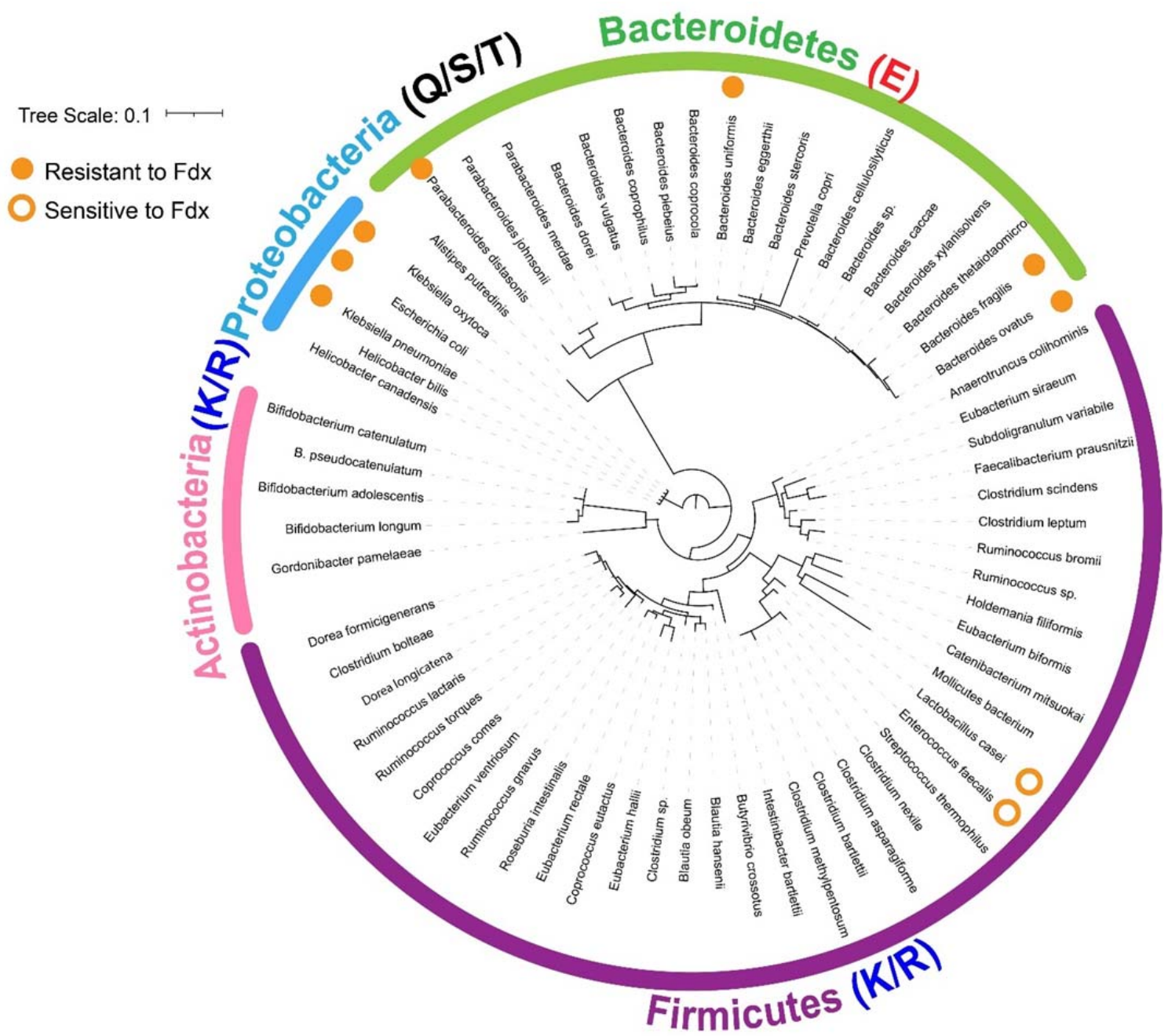

652 Extended Data Fig. 10. Phylogenetic tree demonstrating the clade-specific distribution of the identity of the Fdx-sensitizer. The tree displays the identity of the amino acid corresponding to position $\beta^{\prime} \mathrm{K} 84$ of Cdiff in the most common species from human gut microbiota. Bacterial species were largely picked from ${ }^{28}$ and ${ }^{29}$. The tree was built from 66 small subunit ribosomal RNA sequences by using RaxML ${ }^{52}$ and $\mathrm{iTol}^{53}$. Species with experimentally confirmed resistance ( $\mathrm{MIC}>32 \mu \mathrm{g} / \mathrm{mL}$ ) and sensitivity ( $\mathrm{MIC}<0.125 \mu \mathrm{g} / \mathrm{mL}$ ) to Fdx are marked with solid and open orange circle respectively ${ }^{25}$. The amino acid sequence at $\beta^{\prime} \mathrm{K} 84$ position for corresponding bacteria phyla is denoted by capital letters. The detailed bacterial species are listed in table S4. 

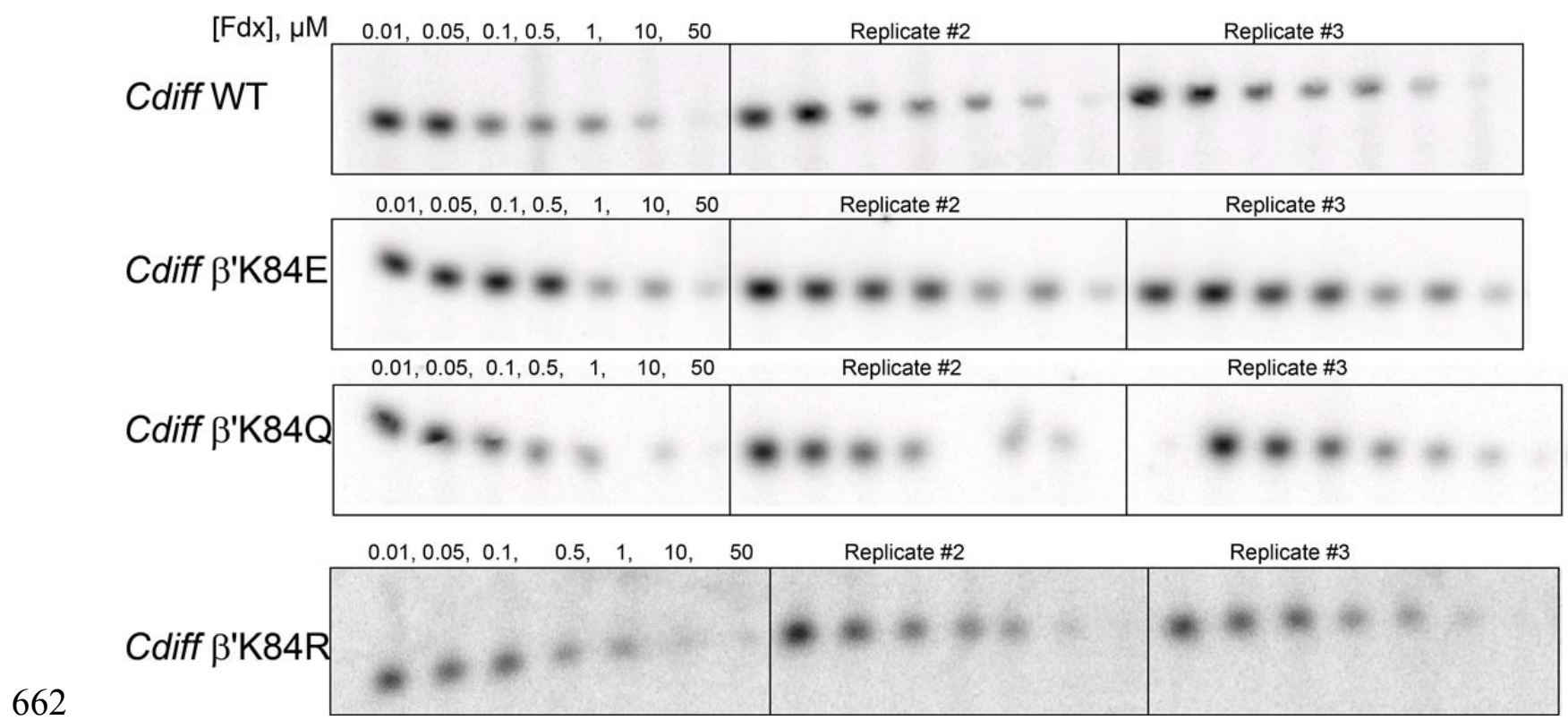

663 Extended Data Fig. 11. In vitro abortive transcription assay measuring Fdx IC50 on Cdiff 664 WT and mutant $\mathbf{E} \boldsymbol{\sigma}^{\mathbf{A}} \mathbf{s}$ related to Fig. 4C. The Cdiff $r r n C$ promoter (Fig. 2C) was used as a 665 template. For each RNAP, three independent experiments were performed for and analyzed on 666 the same gel. 


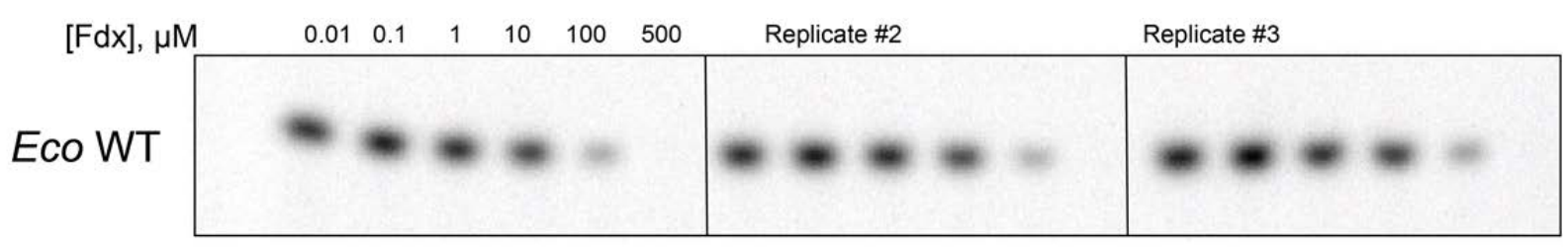
$\begin{array}{llllll}0.01 & 0.1 & 1 & 10 & 100 & 500\end{array}$
Replicate \#2
Replicate \#3

671 Extended Data Fig. 12. In vitro abortive transcription assay measuring Fdx IC50 on Eco

WT and $\boldsymbol{\beta}^{\prime} \mathbf{Q} 94 \mathbf{K} \mathbf{E} \boldsymbol{\sigma}^{70}$ s related to Fig. 4D. The Cdiff rrnC promoter (Fig. 2C) was used as a template. For each RNAP, three independent experiments were performed for and analyzed on

674 the same gel.

675

676 
677 Extended Data Table 1. Antimicrobial efficiency of Fdx on various species.

\begin{tabular}{|l|l|l|}
\hline \multicolumn{1}{|c|}{ Species } & \multicolumn{1}{|c|}{ MIC $(\boldsymbol{\mu g} / \mathbf{m L})$} & \multicolumn{1}{|c|}{$\begin{array}{l}\text { IC50 }(\boldsymbol{\mu M}) \\
\text { in vitro }\end{array}$} \\
\hline C. difficile ATCC17857 & $0.031^{25}$ & 0.2 (this study) \\
\hline M. tuberculosis (H37Rv) & $0.015^{26}$ & $0.21^{7}$ \\
\hline E. coli DH5 $\alpha$ & $>64^{25}$ & $53^{7}$ \\
\hline E. coli D21(rfa, tolC) & $2^{27}$ & N/A \\
\hline B. uniformis ATCC 8492 & $>32^{25}$ & N/A \\
\hline B. ovatus ATCC8483 & $>32^{25}$ & N/A \\
\hline
\end{tabular}


Extended Data Table 2. Cryo-EM data collection, refinement, and validation statistics

681

\begin{tabular}{|c|c|}
\hline & $\begin{array}{l}\text { Cdiff E } \sigma^{\mathrm{A}}+\text { FdG82 } \\
\text { PDB 7L7B }\end{array}$ \\
\hline \multicolumn{2}{|c|}{ Data collection and processing } \\
\hline Magnification & 81,000 \\
\hline Voltage $(\mathrm{kV})$ & 300 \\
\hline $\begin{array}{l}\text { Electron exposure } \\
\left(\mathrm{e}-/ \AA^{2}\right)\end{array}$ & 51 \\
\hline Defocus range $(\mu \mathrm{m})$ & $0.8-2.7$ \\
\hline Pixel size $(\AA)$ & 1.083 \\
\hline Symmetry imposed & $\mathrm{C} 1$ \\
\hline Initial particle images (no.) & $2,502,242$ \\
\hline Final particle images (no.) & 182,390 \\
\hline $\begin{array}{l}\text { Map resolution }(\AA) \\
\text { FSC threshold } 0.143\end{array}$ & 3.26 \\
\hline Map resolution range $(\AA)$ & $2.8-6.6$ \\
\hline \multicolumn{2}{|l|}{ Refinement } \\
\hline Initial models used (PDB code) & $6 \mathrm{BZO}$ \\
\hline Model resolution range $(\AA)$ & $2.7-5.5$ \\
\hline Map sharpening $\mathrm{B}$ factor $\left(\mathrm{A}^{2}\right)$ & 89.9 \\
\hline \multicolumn{2}{|l|}{ Model composition } \\
\hline Non-hydrogen atoms & 24,713 \\
\hline Protein residues & 3125 \\
\hline Ligands & $\begin{array}{l}4\left(\mathrm{Fdx}, \mathrm{Mg}^{2+}, 2\right. \\
\left.\mathrm{Zn}^{2+}\right)\end{array}$ \\
\hline \multicolumn{2}{|l|}{ B factors $\left(A^{2}\right)$} \\
\hline Protein & 131.24 \\
\hline Ligands & 112.25 \\
\hline \multicolumn{2}{|l|}{ R.m.s. deviations } \\
\hline Bond lengths $(\AA)$ & 0.005 \\
\hline Bond angles $\left({ }^{\circ}\right)$ & 0.703 \\
\hline \multicolumn{2}{|l|}{ Validation } \\
\hline MolProbity score & 3.23 \\
\hline Clashscore & 29.04 \\
\hline Poor rotamers $(\%)$ & 10.34 \\
\hline \multicolumn{2}{|l|}{ Ramachandran plot } \\
\hline Favored (\%) & 91.19 \\
\hline Allowed (\%) & 8.81 \\
\hline Outliers (\%) & 0 \\
\hline
\end{tabular}


684 Extended Data Table 3. Complete list of Fdx interacting residues in Cdiff and Mtb RNAP $685 E \sigma^{\mathrm{A}}$ and comparison for those in Eco, B. ovatus, and B. uniformis

\begin{tabular}{|c|c|c|c|c|c|}
\hline Region & M. tuberculosis & C. difficile & E. coli & B. ovatus & B. uniformis \\
\hline SW3 & $\beta-M 1051 \sqrt{ }$ & $\beta$-L1071 V & $\mathrm{L}$ & $\mathrm{L}$ & $\mathrm{L}$ \\
\hline SW3 & $\beta-I 1052 \sqrt{ }$ & $\beta-\mathrm{V} 1072 \sqrt{ }$ & I & I & I \\
\hline SW3 & $\beta-Q 1054 \sqrt{ }$ & $\beta-Q 1074 \sqrt{ }$ & $*$ & $*$ & $*$ \\
\hline SW4 & $\beta-D 1094 \sqrt{ }$ & $\beta-D 1114 \sqrt{ }$ & * & $*$ & $*$ \\
\hline SW4 & $\beta-\mathrm{T} 1096 \sqrt{ }$ & $\beta-V 1116 \sqrt{ }$ & V & V & V \\
\hline SW4 & $\beta-V 1097 \sqrt{ }$ & $\beta-V 1117 \sqrt{ }$ & $\mathrm{N}$ & V & V \\
\hline Clamp & $\beta-V 1100 \mathrm{~V}$ & $\beta-\mathrm{V} 1120 \mathrm{~V}$ & $\mathrm{~T}$ & S & S \\
\hline Clamp & $\beta-\mathrm{K} 1101 \mathrm{~V}$ & $\beta-R 1121 \sqrt{ }$ & K & $\mathrm{K}$ & $\mathrm{K}$ \\
\hline Clamp & $\beta-E 1119 \sqrt{ }$ & $\beta-E 1139 \sqrt{ }$ & $*$ & $*$ & $*$ \\
\hline Clamp & $\beta-S 1120 \sqrt{ }$ & $\beta-S 1140 \sqrt{ }$ & * & $*$ & $*$ \\
\hline ZBD & $\beta^{\prime}-\mathrm{R} 84=\mathrm{HOH} \quad \sqrt{ }$ & $\beta^{\prime}-\mathrm{K} 84 \quad \sqrt{ }$ & Q & $\mathrm{E}$ & $\mathrm{E}$ \\
\hline ZBD & $\beta^{\prime}-A 85 \quad \sqrt{ }$ & $\beta^{\prime}-\mathrm{S} 85 \quad \sqrt{ }$ & $\mathrm{T}$ & $\mathrm{K}$ & $\mathrm{K}$ \\
\hline ZBD & $\beta^{\prime}-K 86 \quad \sqrt{ }$ & $\beta^{\prime}-K 86 \quad \sqrt{ }$ & $*$ & $*$ & $*$ \\
\hline ZBD & $\beta^{\prime}-\mathrm{R} 89 \quad \sqrt{ }$ & $\beta^{\prime}-\mathrm{R} 89 \quad \sqrt{ }$ & * & * & * \\
\hline Lid & $\beta^{\prime}-E 323 \sqrt{ }$ & $\beta^{\prime}-\mathrm{D} 237=\mathrm{HOH} V$ & $\mathrm{D}$ & $\mathrm{E}$ & E \\
\hline Lid & $\beta^{\prime}-\mathrm{L} 324 \sqrt{ }$ & $\beta^{\prime}$-L238 $\sqrt{ }$ & * & $*$ & $*$ \\
\hline Lid & $\beta^{\prime}-\mathrm{P} 326 \sqrt{ }$ & $\beta^{\prime}-\mathrm{P} 240 \sqrt{ }$ & * & $*$ & $*$ \\
\hline Lid & $\beta^{\prime}-\mathrm{S} 338 \sqrt{ }$ & $\beta^{\prime}-\mathrm{S} 252 \sqrt{ }$ & $*$ & $*$ & $*$ \\
\hline SW2 & $\beta^{\prime}-\mathrm{R} 412 \mathrm{~V}$ & $\beta^{\prime}-\mathrm{R} 326 \mathrm{~V}$ & $*$ & $*$ & $*$ \\
\hline$\sigma$-linker & $\sigma-\mathrm{D} 424 \sqrt{ }$ & $\sigma$-E284 V & E & $\mathrm{D}$ & $\mathrm{D}$ \\
\hline RbpA & $\begin{array}{l}\text { R-R10 V } \\
\text { R-E17=HOH V }\end{array}$ & $\begin{array}{l}\text { NA } \\
\text { NA }\end{array}$ & $\begin{array}{l}\text { NA } \\
\text { NA }\end{array}$ & $\begin{array}{l}\text { NA } \\
\text { NA }\end{array}$ & $\begin{array}{l}\text { NA } \\
\text { NA }\end{array}$ \\
\hline 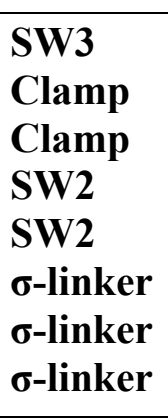 & $\begin{array}{l}\beta-\mathrm{T} 1053 \mathrm{X} \\
\beta^{\prime}-\mathrm{K} 400 \text { (rotamer) X } \\
\beta^{\prime}-\mathrm{D} 404=\mathrm{HOH} \sqrt{ } \\
\beta^{\prime}-\mathrm{L} 405 \mathrm{X} \\
\beta^{\prime}-\mathrm{Q} 415=\mathrm{HOH} \sqrt{ } \\
\sigma-\mathrm{L} 423 \mathrm{X} \\
\sigma-\mathrm{Q} 434 \mathrm{X} \\
\sigma-\mathrm{V} 445 \sqrt{ }\end{array}$ & $\begin{array}{l}\beta-\mathrm{T} 1073 \sqrt{ } \\
\beta^{\prime}-\mathrm{K} 314 \sqrt{ } \\
\beta^{\prime}-\mathrm{D} 318 \mathrm{X} \\
\beta^{\prime}-\mathrm{M} 319 \sqrt{ } \\
\beta^{\prime}-\mathrm{Q} 329 \mathrm{X} \\
\sigma-\mathrm{L} 283 \sqrt{ } \\
\sigma-\mathrm{H} 294=\mathrm{HOH} \sqrt{ } \\
\sigma-\mathrm{P} 305 \mathrm{X}\end{array}$ & $\begin{array}{l}* \\
* \\
* \\
\mathrm{M} \\
* \\
\mathrm{M} \\
\mathrm{H} \\
\mathrm{E}\end{array}$ & $\begin{array}{l}* \\
* \\
* \\
\mathrm{~S} \\
* \\
\mathrm{~V} \\
\mathrm{~S} \\
\mathrm{P}\end{array}$ & $\begin{array}{l}* \\
* \\
* \\
\mathrm{~S} \\
* \\
\mathrm{~V} \\
\mathrm{~S} \\
\mathrm{P}\end{array}$ \\
\hline
\end{tabular}

687 X no corresponding interactions; *, identical residues; grey, similar residues; magenta,

688 distinguished residues 
Extended Data Table 4. List of 66 common bacterial species in human gut profile, related

691 to Fig. 4A. MICs, when reported, are listed. N/A means Not Applicable due to the absence of 692 published values.

\begin{tabular}{|c|c|c|c|}
\hline Species & Phylum & AA at $\boldsymbol{\beta}^{\prime} \mathbf{K 8 4}$ & Published MIC \\
\hline Bacteroides vulgatus & Bacteroidetes & $\mathrm{E}$ & $\mathrm{N} / \mathrm{A}$ \\
\hline Bacteroides uniformis & Bacteroidetes & $\mathrm{E}$ & $32 \mu \mathrm{g} / \mathrm{mL}^{25}$ \\
\hline Bacteroides sp. & Bacteroidetes & $\mathrm{E}$ & N/A \\
\hline Bacteroides xylanisolvens & Bacteroidetes & $\mathrm{E}$ & $\mathrm{N} / \mathrm{A}$ \\
\hline Bacteroides ovatus & Bacteroidetes & $\mathrm{E}$ & $32 \mu \mathrm{g} / \mathrm{ml}^{25}$ \\
\hline $\begin{array}{l}\text { Bacteroides } \\
\text { thetaiotaomicron }\end{array}$ & Bacteroidetes & $\mathrm{E}$ & N/A \\
\hline Bacteroides dorei & Bacteroidetes & $\mathrm{E}$ & $\mathrm{N} / \mathrm{A}$ \\
\hline Parabacteroides distasonis & Bacteroidetes & $\mathrm{E}$ & $32 \mu \mathrm{g} / \mathrm{ml}^{25}$ \\
\hline Parabacteroides merdae & Bacteroidetes & $\mathrm{E}$ & N/A \\
\hline Bacteroides caccae & Bacteroidetes & $\mathrm{E}$ & $\mathrm{N} / \mathrm{A}$ \\
\hline Bacteroides stercoris & Bacteroidetes & $\mathrm{E}$ & $\mathrm{N} / \mathrm{A}$ \\
\hline Bacteroides cellulosilyticus & Bacteroidetes & $\mathrm{E}$ & $\mathrm{N} / \mathrm{A}$ \\
\hline Bacteroides eggerthii & Bacteroidetes & $\mathrm{E}$ & $\mathrm{N} / \mathrm{A}$ \\
\hline Bacteroides plebeius & Bacteroidetes & $\mathrm{E}$ & N/A \\
\hline Bacteroides coprocola & Bacteroidetes & $\mathrm{E}$ & $\mathrm{N} / \mathrm{A}$ \\
\hline Bacteroides coprophilus & Bacteroidetes & $\mathrm{E}$ & $\mathrm{N} / \mathrm{A}$ \\
\hline Bacteroides fragilis & Bacteroidetes & $\mathrm{E}$ & $>32 \mu \mathrm{g} / \mathrm{ml}^{25}$ \\
\hline Alistipes putredinis & Bacteroidetes & $\mathrm{E}$ & $\mathrm{N} / \mathrm{A}$ \\
\hline Parabacteroides johnsonii & Bacteroidetes & $\mathrm{E}$ & $\mathrm{N} / \mathrm{A}$ \\
\hline Prevotella copri & Bacteroidetes & $\mathrm{E}$ & $\mathrm{N} / \mathrm{A}$ \\
\hline Anaerotruncus colihominis & Firmicutes & $\mathrm{R}$ & $\mathrm{N} / \mathrm{A}$ \\
\hline Blautia hansenii & Firmicutes & $\mathrm{K}$ & $\mathrm{N} / \mathrm{A}$ \\
\hline Blautia obeum & Firmicutes & $\mathrm{K}$ & $\mathrm{N} / \mathrm{A}$ \\
\hline Butyrivibrio crossotus & Firmicutes & $\mathrm{K}$ & $\mathrm{N} / \mathrm{A}$ \\
\hline Catenibacterium mitsuokai & Firmicutes & $\mathrm{R}$ & N/A \\
\hline Intestinibacter bartlettii & Firmicutes & $\mathrm{K}$ & $\mathrm{N} / \mathrm{A}$ \\
\hline Clostridium bolteae & Firmicutes & $\mathrm{K}$ & $\mathrm{N} / \mathrm{A}$ \\
\hline Clostridium leptum & Firmicutes & $\mathrm{R}$ & N/A \\
\hline Clostridium methylpentosum & Firmicutes & $\mathrm{R}$ & $\mathrm{N} / \mathrm{A}$ \\
\hline Clostridium scindens & Firmicutes & $\mathrm{K}$ & N/A \\
\hline Clostridium sp. & Firmicutes & $\mathrm{K}$ & $\mathrm{N} / \mathrm{A}$ \\
\hline Clostridium nexile & Firmicutes & $\mathrm{K}$ & $\mathrm{N} / \mathrm{A}$ \\
\hline Clostridium asparagiforme & Firmicutes & $\mathrm{K}$ & N/A \\
\hline Clostridium bartlettii & Firmicutes & $\mathrm{K}$ & $\mathrm{N} / \mathrm{A}$ \\
\hline Coprococcus comes & Firmicutes & $\mathrm{K}$ & $\mathrm{N} / \mathrm{A}$ \\
\hline Coprococcus eutactus & Firmicutes & $\mathrm{K}$ & N/A \\
\hline Dorea formicigenerans & Firmicutes & $\mathrm{K}$ & $\mathrm{N} / \mathrm{A}$ \\
\hline Dorea longicatena & Firmicutes & $\mathrm{K}$ & $\mathrm{N} / \mathrm{A}$ \\
\hline Enterococcus faecalis & Firmicutes & $\mathrm{R}$ & $4 \mu \mathrm{g} / \mathrm{mL}^{25}$ \\
\hline
\end{tabular}




\begin{tabular}{|c|c|c|c|}
\hline $\begin{array}{l}\text { Eubacterium(Holdemanella) } \\
\text { biforme }\end{array}$ & Firmicutes & $\mathrm{K}$ & $\mathrm{N} / \mathrm{A}$ \\
\hline Eubacterium hallii & Firmicutes & $\mathrm{K}$ & $\mathrm{N} / \mathrm{A}$ \\
\hline Eubacterium rectale & Firmicutes & $\mathrm{K}$ & $\mathrm{N} / \mathrm{A}$ \\
\hline Eubacterium siraeum & Firmicutes & $\mathrm{R}$ & $\mathrm{N} / \mathrm{A}$ \\
\hline Eubacterium ventriosum & Firmicutes & $\mathrm{K}$ & $\mathrm{N} / \mathrm{A}$ \\
\hline Faecalibacterium prausnitzii & Firmicutes & $\mathrm{K}$ & $\mathrm{N} / \mathrm{A}$ \\
\hline Holdemania filiformis & Firmicutes & $\mathrm{K}$ & $\mathrm{N} / \mathrm{A}$ \\
\hline Lactobacillus casei & Firmicutes & $\mathrm{R}$ & $1 \mu \mathrm{g} / \mathrm{mL}^{25}$ \\
\hline Mollicutes bacterium & Firmicutes & $\mathrm{R}$ & $\mathrm{N} / \mathrm{A}$ \\
\hline Roseburia intestinalis & Firmicutes & $\mathrm{K}$ & $\mathrm{N} / \mathrm{A}$ \\
\hline Ruminococcus bromii & Firmicutes & $\mathrm{R}$ & $\mathrm{N} / \mathrm{A}$ \\
\hline Ruminococcus gnavus & Firmicutes & $\mathrm{K}$ & $\mathrm{N} / \mathrm{A}$ \\
\hline Ruminococcus lactaris & Firmicutes & $\mathrm{K}$ & $\mathrm{N} / \mathrm{A}$ \\
\hline Ruminococcus sp & Firmicutes & $\mathrm{K}$ & $\mathrm{N} / \mathrm{A}$ \\
\hline Ruminococcus torques & Firmicutes & $\mathrm{K}$ & $\mathrm{N} / \mathrm{A}$ \\
\hline Streptococcus thermophilus & Firmicutes & $\mathrm{R}$ & N/A \\
\hline Subdoligranulum variabile & Firmicutes & $\mathrm{R}$ & N/A \\
\hline Bifidobacterium adolescentis & Actinobacteria & $\mathrm{K}$ & $\mathrm{N} / \mathrm{A}$ \\
\hline Bifidobacterium catenulatum & Actinobacteria & $\mathrm{K}$ & $\mathrm{N} / \mathrm{A}$ \\
\hline Bifidobacterium longum & Actinobacteria & $\mathrm{K}$ & $\mathrm{N} / \mathrm{A}$ \\
\hline $\begin{array}{l}\text { Bifidobacterium } \\
\text { pseudocatenulatum }\end{array}$ & Actinobacteria & $\mathrm{K}$ & $\mathrm{N} / \mathrm{A}$ \\
\hline Gordonibacter pamelaeae & Actinobacteria & $\mathrm{R}$ & $\mathrm{N} / \mathrm{A}$ \\
\hline Escherichia coli & Proteobacteria & Q & $>32 \mu \mathrm{g} / \mathrm{mL}^{25}$ \\
\hline Klebsiella pneumoniae & Proteobacteria & Q & $>32 \mu \mathrm{g} / \mathrm{mL}^{25}$ \\
\hline Klebsiella oxytoca & Proteobacteria & Q & $64 \mu \mathrm{g} / \mathrm{mL}^{25}$ \\
\hline Helicobacter bilis & Proteobacteria & $\mathrm{T}$ & $\mathrm{N} / \mathrm{A}$ \\
\hline Helicobacter canadensis & Proteobacteria & $\mathrm{S}$ & $\mathrm{N} / \mathrm{A}$ \\
\hline
\end{tabular}

